\title{
An Amplitude Equation Approach to Contextual Effects in Visual Cortex
}

\author{
Paul C. Bressloff \\ bressloff@math.utah.edu \\ Department of Mathematics, University of Utah, Salt Lake City, Utah 84112, U.S.A.
}

\section{Jack D. Cowan}

cowan@math.uchicago.edu

Mathematics Department, University of Chicago, Chicago, IL 60637, U.S.A.

A mathematical theory of interacting hypercolumns in primary visual cortex (V1) is presented that incorporates details concerning the anisotropic nature of long-range lateral connections. Each hypercolumn is modeled as a ring of interacting excitatory and inhibitory neural populations with orientation preferences over the range 0 to 180 degrees. Analytical methods from bifurcation theory are used to derive nonlinear equations for the amplitude and phase of the population tuning curves in which the effective lateral interactions are linear in the amplitudes. These amplitude equations describe how mutual interactions between hypercolumns via lateral connections modify the response of each hypercolumn to modulated inputs from the lateral geniculate nucleus; such interactions form the basis of contextual effects. The coupled ring model is shown to reproduce a number of orientation-dependen $t$ and contrast-dependent features observed in center-surround experiments. A major prediction of the model is that the anisotropy in lateral connections results in a nonuniform modulatory effect of the surround that is correlated with the orientation of the center.

\section{Introduction}

The discovery that a majority of neurons in the visual or striate cortex of cats and primates (usually referred to as V1) respond selectively to the local orientation of visual contrast patterns (Hubel \& Wiesel, 1962) initiated many studies of the precise circuitry underlying this property. Two cortical circuits have been fairly well characterized. There is a local circuit operating at subhypercolumn dimensions $(<0.7 \mathrm{~mm})$ in monkeys comprising strong orientation-specific recurrent excitation and weaker intrahypercolumnar inhibition (Michalski, Gerstein, Czarkowska, \& Tarnecki, 1983; Hata, Tsumoto, Sato, Hagihara, \& Tamura, 1988; Douglas, Koch, Mahowald, Martin, \& Suarez, 1995). The other circuit operates between hypercolumns, 
connecting cells with similar orientation preferences separated by up to several millimeters of cortical tissue (Gilbert \& Wiesel, 1989; Hirsch \& Gilbert, 1992; Malach, Amir, Harel, \& Grinvald, 1993; Fitzpatrick, Zhang, Schofield, \& Muly, 1993; Grinvald, Lieke, Frostig, \& Hildesheim, 1994; Yoshioka, Blasdel, Levitt, \& Lund, 1996). The lateral connections that mediate this circuit arise almost exclusively from excitatory neurons (Rockland \& Lund, 1983; Gilbert \& Wiesel, 1983), although 20\% terminate on inhibitory cells and can thus have significant inhibitory effects (McGuire, Gilbert, Rivlin, \& Wiesel, 1991).

Stimulation of a hypercolumn via lateral connections modulates rather than initiates spiking activity (Hirsch \& Gilbert, 1992; Toth, Rao, Kim, Somers, \& Sur, 1996). Thus, the lateral connectivity is ideally structured to provide local cortical processes with contextual information about the global nature of stimuli. As a consequence, these lateral connections have been invoked to explain a wide variety of context-dependent visual processing phenomena (Gilbert, Das, Ito, Kapadia, \& Westheimer, 1996; Fitzpatrick, 2000). (Interestingly, contextual processing also occurs in extrastriate visual areas, and feedback from these areas to V1 has been invoked to explain aspects of contextual processing; Wenderoth \& Johnstone, 1988). One common experimental paradigm is to investigate the response to stimuli consisting of circular (center) and annular (surround) gratings of differing contrasts, orientations, and diameters. When both center and surround are stimulated with strong suprathreshold inputs, one typically finds that the surround suppresses the center unit's tuning response for surround stimulation close to the orientation of the unit's peak tuning response and facilitates the response when the surround is stimulated at orientations sufficiently dissimilar to the preferred orientation of the center (Blakemore \& Tobin, 1972; Li \& Li, 1994; Sillito, Grieve, Jones, Cudeiro, \& Davis, 1995).

The center response to surround stimulation depends significantly on the contrast of center stimulation (Toth et al., 1996; Levitt \& Lund, 1997; Polat, Mizobe, Pettet, Kasamatsu, \& Norcia, 1998). For example, a fixed surround stimulus tends to facilitate responses to stimuli at a preferred orientation when the center contrast is low but suppresses responses when it is high. Both effects are strongest when center and surround stimuli are iso-oriented. The response to variations in the size of a stimulus is also contrast dependent. For example, responses to a high-contrast circular grating decline beyond a characteristic preferred stimulus size due to activation of an inhibitory surround. In addition, the effective size of the excitatory receptive field tends to increase with decreasing contrast, and at low contrasts becomes a monotonically increasing function of stimulus size (Jagadeesh \& Ferster, 1990; Sceniak, Ringach, Hawken, \& Shapley, 1999).

One common approach to modeling the role of lateral connections in center-surround modulation is to consider a reduced local cortical circuit composed of excitatory and inhibitory populations receiving feedforward 
inputs from the lateral geniculate nucleus (LGN), together with long-range intracortical inputs, some of which could provide feedback from higher cortical areas (Somers, Nelson, \& Sur, 1994; Mundel, 1996; Mundel, Dimitrov, \& Cowan, 1997; Somers et al., 1998; Li, 1999; Dragoi \& Sur, 2000; Stetter, Bartsch, \& Obermayer, 2000; Grossberg \& Raizada, 2000). Inclusion of some form of contrast-related asymmetry between local excitatory and inhibitory neurons is then sufficient to account for the switch between low-contrast facilitation and high-contrast suppression (Somers et al., 1998; Stetter et al., 2000). Although these (mainly) computational studies have provided some insights into the possible role of lateral connections in mediating center-surround effects, a more general analytical framework has so far been lacking.

In this article, we develop such a framework by considering the nonlinear dynamics of interacting hypercolumns. In particular, previous work on the dynamics of sharp orientation tuning in recurrent models of a cortical hypercolumn (Ben-Yishai, Bar-Or, \& Sompolinsky, 1995; Somers, Nelson, \& Sur, 1995; Vidyasagar, Pei, \& Volgushev, 1996; Ben-Yishai, Hansel, \& Sompolinsky, 1997; Mundel et al., 1997; Bressloff, Bressloff, \& Cowan, 2000; Pugh, Ringach, Shapley, \& Shelley, 2000) is extended to incorporate the effects of lateral connections between hypercolumns. A related computational approach has been initiated by McLaughlin, Shapley, Shelley, and Wielaard (2000). Our basic assumptions are as follows: (1) each active hypercolumn is close to a bifurcation point signaling the onset of sharp orientation tuning, and (2) the interactions between hypercolumns are weak. We first use analytical methods from bifurcation theory to derive nonlinear equations for the amplitude and phase of the tuning curves in which the effective interactions are linear in the amplitudes. (Amplitude equations with linear interaction terms also arise in studies of weakly interacting neurons; Hoppensteadt \& Izhikevich, 1997). These amplitude equations describe how mutual interactions between hypercolumns via lateral connections modify the response of each hypercolumn to modulated inputs from the lateral geniculate nucleus; such interactions form the basis of contextual effects. We then show how our model reproduces a number of orientationdependent and contrast-dependent features observed in center-surround experiments.

One novel aspect of our model is that we incorporate the explicit anisotropy of lateral connections as observed in a number of optical imaging experiments (Yoshioka et al., 1996; Bosking, Zhang, Schofield, \& Fitzpatrick, 1997). In recent studies of the global dynamics of V1, idealized as a continuous two-dimensional sheet of interacting hypercolumns, we showed that the patterns of connection exhibit a very interesting symmetry (Wiener, 1994; Cowan, 1997; Bressloff, Cowan, Golubitsky, Thomas, \& Wiener, 2001a); they are invariant under the action of the planar Euclidean group E(2) the group of rigid motions in the plane-rotations, reflections, and translations. By virtue of the anisotropy of the lateral connections, they are also 
invariant with respect to certain shifts and twists of the plane such that a new $\mathbf{E}(2)$ group action is needed to represent its properties. This shift-twist symmetry plays an important role in determining which cortical activity patterns form through spontaneous symmetry breaking. Our previous results on the global dynamics of V1 can also be obtained by considering a continuum version of the amplitude equations for weakly interacting hypercolumns derived in this paper. This establishes that both the feedforward response properties of $\mathrm{V} 1$ and its intrinisic dynamical properties can be understood within the same analytical framework.

\section{A Dynamical Model of V1 and Its Intrinsic Circuitry}

Recent work using optical imaging (Blasdel \& Salama, 1986; Blasdel, 1992) augments the early work of Hubel and Wiesel (1962) concerning the largescale organization of iso-orientation patches in V1. It shows that approximately every $0.7 \mathrm{~mm}$ or so in monkey $\mathrm{V} 1$, there is an iso-orientation patch of a given preference $\phi$. We therefore view the tangential structure of the cortex as a lattice of hypercolumns, where each hypercolumn comprises a continuum of iso-orientation patches that are designated by their preferred orientation $\phi \in\left[-90^{\circ}, 90^{\circ}\right]$. This simplified anatomy is most easily motivated (though the analysis does not depend on this assumption in any crucial manner) if we assume a pinwheel two-dimensional architecture for V1 (Obermayer \& Blasdel, 1993; Bonhoeffer, Kim, Malonek, Shoham, \& Grinvald, 1995). The sharply orientation-tuned elements of a hypercolumn then form a ring of interacting neural populations. (A lattice model is also consistent with the observation that each location in the visual field is represented in V1, roughly speaking, by a hypercolumn-sized region containing all orientations.) In addition to this two-dimensional tangential architecture, V1 has a complex laminar vertical structure. Although this vertical organization has been studied in some detail, its functional characteristics with regard to orientation selectivity are largely unknown. The solution we adopt here is to collapse this vertical organization into the dynamical interaction between two neuronal components: one excitatory $(E)$, representing pyramidal cells, and the other inhibitory $(I)$. The latter lumps together a number of different types of interneuron, for example, interneurons with horizontally distributed axonal fields of the basket cell subtype and inhibitory interneurons that have predominantly vertically aligned axonal fields (see Figure 1).

Suppose that there are $N$ hypercolumns labeled $i=1, \ldots, N$. Let $a_{l}\left(\mathbf{r}_{i}, \phi, t\right)$ denote the average membrane potential or activity at time $t$ of a population of cells of type $l=E, I$ belonging to an iso-orientation patch of the $i$ th hypercolumn, $i=1, \ldots, N$, where $\mathbf{r}_{i}$ denotes the cortical position of the hypercolumn (on some coarsely grained spatial scale) and $\phi \in\left[-90^{\circ}, 90^{\circ}\right]$ is the orientation preference of the patch. The activity variables $a_{l}$ evolve to 


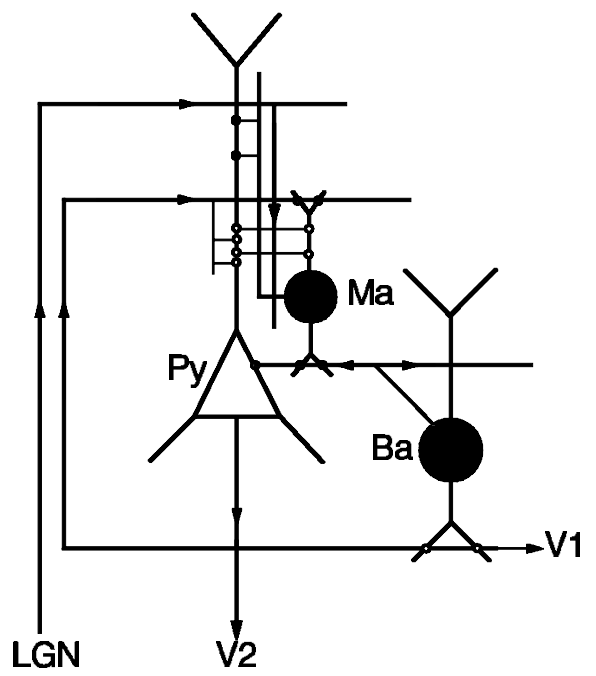

Figure 1: Local interacting neuronal populations. Py-excitatory population of pyramidal cells; Ba-spatially extended basket cell inhibitory population; Ma-local inhibitory population; Martinotti, chandelier, and double bouquet populations.

the set of equations

$$
\begin{aligned}
& \zeta \frac{\partial a_{l}\left(\mathbf{r}_{i}, \phi, t\right)}{\partial t}=-a_{l}\left(\mathbf{r}_{i}, \phi, t\right)+h_{l}\left(\mathbf{r}_{i}, \phi\right) \\
& \quad+\sum_{m=E, I} \sum_{j=1}^{N} \int_{-\pi / 2}^{\pi / 2} w_{l m}\left(\mathbf{r}_{i}, \phi \mid \mathbf{r}_{j}, \phi^{\prime}\right) \times \sigma_{m}\left(a_{m}\left(\mathbf{r}_{j}, \phi^{\prime}, t\right)\right) \frac{d \phi^{\prime}}{\pi}
\end{aligned}
$$

where $\zeta$ is a decay time constant (set equal to unity), $h_{l}\left(\mathbf{r}_{i}, \phi\right)$ is the input to the $i$ th hypercolumn (in this study presumed to be from the LGN), and $\sigma$ is taken to be a smooth output function, ${ }^{1}$

$$
\sigma(x)=\frac{1}{1+\mathrm{e}^{-\eta_{l}\left(x-\kappa_{l}\right)}},
$$

${ }^{1}$ If $\sigma_{l}$ is interpreted as the firing rate of a single neuron with $a_{l}$ its membrane potential, then $\sigma_{l}$ typically has a hard threshold, that is, $\sigma_{l}\left(a_{l}\right)=0$ for $a_{l}<\eta_{l}$. However, at the population level, such a firing-rate function can be smoothed out by either spontaneous activity or dispersion of membrane properties within the population (Wilson \& Cowan, 1972). From a mathematical perspective, the assumption of smoothness is needed in order to carry out the bifurcation analysis of Section 3. 
where $\eta_{l}$ determines the slope or sensitivity of the input-output characteristics of the population and $\kappa_{l}$ is a threshold. The kernel $w_{l m}\left(\mathbf{r}_{i}, \phi \mid \mathbf{r}_{j}, \phi^{\prime}\right)$ represents the strength or weight of connections from the $m$ th population of the iso-orientation patch $\phi^{\prime}$ at cortical position $\mathbf{r}_{j}$ to the $l$ th population of the orientation patch $\phi$ at position $\mathbf{r}_{i}$. Note that in this article, we do not specify how visual stimuli impinging on the retina are mapped to the cortex via the LGN. We simply assume that when there is an oriented stimulus in the (aggregate) classical receptive field of the $i$ th hypercolumn, then the input $h\left(\mathbf{r}_{i}, \phi\right)$ is maximal at $\phi=\Phi_{i}$ where $\Phi_{i}$ is the stimulus orientation. We also assume that the hypercolumns have nonoverlapping aggregate fields so that each probes a distinct region of visual space.

Optical imaging combined with labeling techniques has generated considerable information concerning the pattern of connections both within and between hypercolumns (Blasdel \& Salama, 1986; Blasdel, 1992; Malach et al., 1993; Yoshioka et al., 1996; Bosking et al., 1997). A particularly striking result concerns the intrinsic lateral connections in layers II, III, and (to some extent) V of V1. The axons of these connections make terminal arbors only every $0.7 \mathrm{~mm}$ or so along their tracks (Rockland \& Lund, 1983; Gilbert \& Wiesel, 1983), and they seem to connect mainly to cells with similar orientation preferences (Malach et al., 1993; Yoshioka et al., 1996; Bosking et al., 1997). In addition, there is a pronounced anisotropy of the pattern of such connections; its long axis runs parallel to a patch's preferred orientation (Gilbert \& Wiesel, 1983; Bosking et al., 1997). Thus, differing iso-orientation patches connect to patches in neighboring hypercolumns in differing directions. This contrasts with the pattern of connectivity within any one hypercolumn that is much more isotropic; any given iso-orientation patch connects locally in all directions to all neighboring patches within a radius of less than $0.7 \mathrm{~mm}$.

Motivated by these observations concerning the intrinsic circuitry of V1, we decompose $w$ in terms of local connections from elements within the same hypercolumn and patchy (excitatory) lateral connections from elements in other hypercolumns:

$$
w_{l m}\left(\mathbf{r}_{i}, \phi \mid \mathbf{r}_{j}, \phi^{\prime}\right)=w_{l m}\left(\phi-\phi^{\prime}\right) \delta_{i, j}+\epsilon \beta_{l} \delta_{m, E} \hat{w}\left(\mathbf{r}_{i}, \phi \mid \mathbf{r}_{j}, \phi^{\prime}\right)\left(1-\delta_{i, j}\right),
$$

where $\epsilon$ is a parameter that measures the weight of lateral relative to local connections. Observations by Hirsch and Gilbert (1992) suggest that $\epsilon$ is small and therefore that the lateral connections modulate rather than drive V1 activity. The relative strengths of the lateral inputs into local excitatory and inhibitory populations are represented by the factors $\beta_{l}$. (Recall that although the lateral connections are excitatory-Rockland \& Lund, 1983; Gilbert \& Wiesel, 1983-20\% of the connections in layers II and III of V1 end on inhibitory interneurons, so the overall action of the lateral connections can become inhibitory, especially at high levels of activity; Hirsch \& Gilbert, 1992). The contrast dependence of the factors $\beta_{l}$ will be considered 
in Section 4. Substituting equation 2.3 into equation 2.1 gives

$$
\begin{aligned}
& \frac{\partial a_{l}\left(\mathbf{r}_{i}, \phi, t\right)}{\partial t}=-a_{l}\left(\mathbf{r}_{i}, \phi, t\right)+\sum_{m=E, I} \int_{-\pi / 2}^{\pi / 2} w_{l m}\left(\phi-\phi^{\prime}\right) \sigma_{m}\left(a_{m}\left(\mathbf{r}_{i}, \phi^{\prime}, t\right)\right) \\
& \frac{d \phi^{\prime}}{\pi}+h_{l}\left(\mathbf{r}_{i}, \phi\right)+\epsilon \beta_{l} \sum_{j \neq i} \int_{-\pi / 2}^{\pi / 2} \hat{w}\left(\mathbf{r}_{i}, \phi \mid \mathbf{r}_{j}, \phi^{\prime}\right) \sigma_{E}\left(a_{E}\left(\mathbf{r}_{j}, \phi^{\prime}, t\right)\right) \frac{d \phi^{\prime}}{\pi}
\end{aligned}
$$

for $i=1, \ldots, N$.

In order to incorporate information concerning the anisotropic nature of lateral connections, we further decompose $\hat{w}$ according to ${ }^{2}$

$$
\hat{w}\left(\mathbf{r}_{i}, \phi \mid \mathbf{r}_{j}, \phi^{\prime}\right)=\hat{w}\left(r_{i j}\right) p_{0}\left(\phi^{\prime}-\theta_{i j}\right) p_{1}\left(\phi-\phi^{\prime}\right),
$$

where $\mathbf{r}_{j}=\mathbf{r}_{i}+r_{i j}\left(\cos \left(\theta_{i j}\right), \sin \left(\theta_{i j}\right)\right)$. In the special case $p_{k}(\phi)=\delta(\phi)$, the distribution $\hat{w}\left(r_{i j}\right)$ determines the weight of lateral connections between iso-orientation patches separated by a cortical distance $r_{i j}$ along a visuotopic axis whose direction $\theta_{i j}$ is parallel to their (common) orientation preference (see Figure 2). The $\pi$-periodic functions $p_{k}(\phi)$ then determine the spread of the lateral connections with respect to the visuotopic axis for $k=0$ (see Figure 3a) and orientation preference for $k=1$ (see Figure 3b). We take $p_{k}(\phi)$ to be an even, monotonically decreasing function of $\phi$ over the domain $-\pi / 2<\phi<\pi / 2$ such that $\int_{-\pi / 2}^{\pi / 2} p_{k}(\phi) d \phi / \pi=1$.

\section{Amplitude Equation for Interacting Hypercolumns}

3.1 Sharp Orientation Tuning in a Local Cortical Circuit. In the absence of lateral connections $(\epsilon=0)$, each hypercolumn is independently described by the ring model of orientation tuning (Somers et al., 1995; Ben-Yishai et al., 1995, 1997; Mundel et al., 1997; Bressloff et al., 2000). That is, equation 2.4 reduces to the pair of equations

$$
\begin{gathered}
\frac{\partial a_{E}}{\partial t}=-a_{E}+w_{E E} * \sigma_{E}\left(a_{E}\right)-w_{E I} * \sigma_{I}\left(a_{I}\right)+h_{E} \\
\frac{\partial a_{I}}{\partial t}=-a_{I}+w_{I E} * \sigma_{E}\left(a_{E}\right)-w_{I I} * \sigma\left(a_{I}\right)+h_{I},
\end{gathered}
$$

where $*$ indicates a convolution operation,

$$
w * f\left(\mathbf{r}_{i}, \phi\right)=\int_{-\pi / 2}^{\pi / 2} w\left(\phi-\phi^{\prime}\right) f\left(\mathbf{r}_{i}, \phi^{\prime}\right) \frac{d \phi^{\prime}}{\pi},
$$

2 The distribution of lateral connections given by equation 2.5 differs from the one used by $\mathrm{Li}$ (1999) in that there is no explicit cross-orientation inhibition. 


\section{HYPERCOLUMNS}

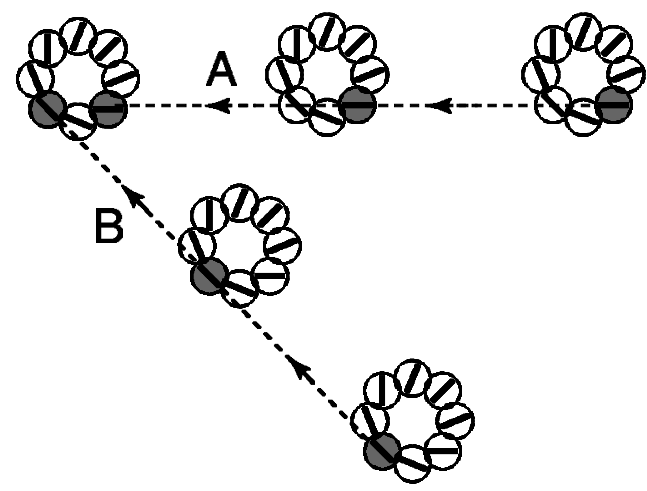

Figure 2: Anisotropic lateral interactions connect iso-orientation patches located along a visuotopic axis parallel to their (common) orientation preference. Horizontal patches are connected along axis A, whereas oblique patches are connected along axis B.

for a hypercolumn at a fixed cortical position $\mathbf{r}_{i}$. In the case of $\phi$-independent external inputs, $h_{l}\left(\mathbf{r}_{i}, \phi\right)=\bar{h}_{l}\left(\mathbf{r}_{i}\right), i=1, \ldots, N$, there exists at least one fixedpoint solution $a_{l}\left(\mathbf{r}_{i}, \phi\right)=\bar{a}_{l}\left(\mathbf{r}_{i}\right)$ of equations 3.1 and 3.2, which satisfies the algebraic equations

$$
\begin{aligned}
\bar{a}_{E} & =W_{E E}(0) \sigma_{E}\left(\bar{a}_{E}\right)-W_{E I}(0) \sigma_{I}\left(\bar{a}_{I}\right)+\bar{h}_{E} \\
\bar{a}_{I} & =W_{I E}(0) \sigma_{E}\left(\bar{a}_{E}\right)-W_{I I}(0) \sigma_{I}\left(\bar{a}_{I}\right)+\bar{h}_{I},
\end{aligned}
$$
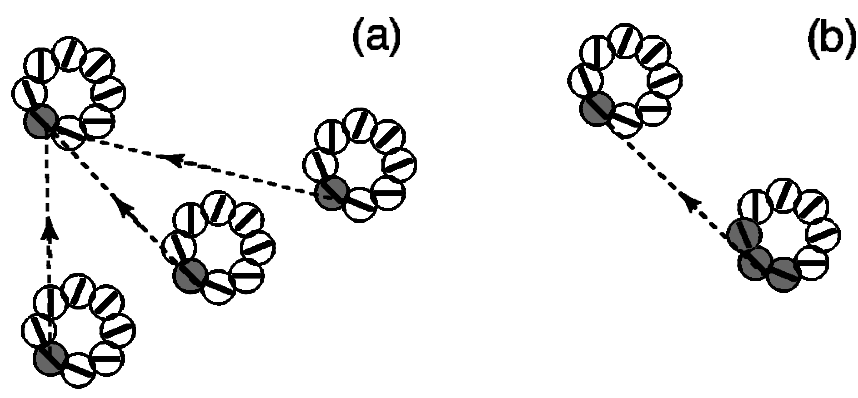

Figure 3: Angular spread in the anisotropic lateral connections with respect to the (a) visuotopic axis and (b) orientation preference. Shaded orientation patches are connected. 
where $W_{l m}(0)=\int_{-\pi / 2}^{\pi / 2} w_{l m}(\phi) d \phi / \pi$. If $\bar{h}_{l}$ is sufficiently small relative to the threshold $\kappa_{l}$, then this fixed point is unique and stable for each $\mathbf{r}_{i}$. Under the change of coordinates $a_{l} \rightarrow a_{l}-\bar{h}_{l}$, it can be seen that the effect of $\bar{h}_{l}$ is to shift the threshold by the amount $-\bar{h}_{l}$. Thus, there are two ways to increase the excitability of the network and thus destabilize the fixed point: increasing the external input $\bar{h}_{l}$ or reducing the threshold $\kappa_{l}$. In the case of external visual stimuli, adaptation mechanisms tend to filter out the uniform part of the stimulus so that the contribution to $\bar{h}_{l}$ from the LGN is expected to be small. We assume, however, that various arousal and attentional mechanism act so that neurons sit close to threshold and can respond to small spatial (and temporal) variations of $\bar{h}_{l}$ (Tsodyks \& Sejnowski, 1995). An alternative mechanism for raising the excitability of the cortex is through the action of drugs on certain brain stem nuclei, which can induce the experience of seeing geometric visual hallucinations (Ermentrout \& Cowan, 1979; Bressloff et al., 2001a; Bressloff, Cowan, Golubitsky, Thomas, \& Wiener, 2001b).

The local stability of $\left(\bar{a}_{E}, \bar{a}_{I}\right)$ is found by linearization:

$$
\begin{aligned}
& \frac{\partial b_{E}}{\partial t}=-b_{E}+\left[\sigma_{E}^{\prime}\left(\bar{a}_{E}\right) w_{E E} * b_{E}-\sigma_{I}^{\prime}\left(\bar{b}_{I}\right) w_{E I} * b_{I}\right] \\
& \frac{\partial b_{I}}{\partial t}=-b_{I}+\left[\sigma_{E}^{\prime}\left(\bar{a}_{E}\right) w_{I E} * b_{E}-\sigma_{I}^{\prime}\left(\bar{a}_{I}\right) w_{I I} * b_{I}\right],
\end{aligned}
$$

where $b_{l}\left(\mathbf{r}_{j}, \phi, t\right)=a_{l}\left(\mathbf{r}_{j}, \phi, t\right)-\bar{a}_{l}\left(\mathbf{r}_{j}\right)$ and $\sigma_{l}^{\prime}(x)=d \sigma(x) / d x$. Equations 3.6 and 3.7 have solutions of the form

$$
b_{l}\left(\mathbf{r}_{j}, \phi, t\right)=B_{l} \mathrm{e}^{\lambda t}\left[z\left(\mathbf{r}_{j}\right) \mathrm{e}^{2 i n \phi}+\bar{z}\left(\mathbf{r}_{j}\right) \mathrm{e}^{-2 i n \phi}\right],
$$

where $z\left(\mathbf{r}_{j}\right)$ is an arbitrary (complex) amplitude. For each positive integer $n$, the eigenvalues $\lambda$ and corresponding eigenvectors $\mathbf{B}=\left(B_{E}, B_{I}\right)^{\mathrm{T}}$ satisfy the matrix equation

$$
(1+\lambda) \mathbf{B}=\left(\begin{array}{ll}
\sigma_{E}^{\prime}\left(\bar{a}_{E}\right) W_{E E}(n) & -\sigma_{I}^{\prime}\left(\bar{a}_{I}\right) W_{E I}(n) \\
\sigma_{E}^{\prime}\left(\bar{a}_{E}\right) W_{I E}(n) & -\sigma_{I}^{\prime}\left(\bar{a}_{I}\right) W_{I I}(n)
\end{array}\right) \mathbf{B},
$$

where $W_{l m}(n)$ is the $n$th Fourier coefficient in the expansion of the $\pi$-periodic weights kernels $w_{l m}(\phi)$,

$$
w_{l m}(\phi)=W_{l m}(0)+2 \sum_{n=1}^{\infty} W_{l m}(n) \cos (2 n \phi), \quad l, m=E, I,
$$

and we assume that $w_{l m}(\phi)=w_{l m}(-\phi)$.

For the sake of illustration, suppose that $\sigma_{m}^{\prime}\left(\bar{a}_{m}\right)=\mu$, so that an increase in the excitability of the network can be modeled as an increase in $\mu$. Equation 3.9 then has solutions of the form

$$
\lambda_{n}^{ \pm}=-1+\mu W_{n}^{ \pm}
$$


for integer $n$, where

$$
W_{n}^{ \pm}=\frac{1}{2}\left[W_{E E}(n)-W_{I I}(n) \pm \Sigma(n)\right]
$$

are the eigenvalues of the weight matrix with

$$
\Sigma(n)=\sqrt{\left[W_{E E}(n)+W_{I I}(n)\right]^{2}-4 W_{E I}(n) W_{I E}(n)} .
$$

The corresponding eigenvectors (up to an arbitrary normalization) are

$$
\mathbf{B}_{n}^{ \pm}=\left(\begin{array}{c}
W_{E I}(n) \\
\frac{1}{2}\left[W_{E E}(n)+W_{I I}(n) \mp \Sigma(n)\right]
\end{array}\right) .
$$

Suppose that $\operatorname{Re} W_{n}^{+}$has a unique maximum as a function of $n \geq 0$ at $n=1$, with $\operatorname{Im} W_{1}^{+}=0$, and define $\mu_{c}=1 / W_{1}^{+}$. For $\mu<\mu_{c}$, we have $\lambda_{n}^{ \pm}<0$ for all $n$, and thus the homogeneous resting state is stable. However, as $\mu$ is steadily increased, the homogeneous state will destabilize at the critical point $\mu=\mu_{c}$ due to excitation of eigenmodes of the form $\mathbf{B}_{1}^{+}\left[z\left(\mathbf{r}_{j}\right) \mathrm{e}^{2 i \phi}+\bar{z}\left(\mathbf{r}_{j}\right) \mathrm{e}^{-2 i \phi}\right]$, where $z\left(\mathbf{r}_{j}\right)$ is an arbitrary complex function of $\mathbf{r}$. It can be shown that the saturating nonlinearities of the system stabilize the tuning curves beyond the critical point $\mu_{c}$ (Ermentrout, 1998; Bressloff et al., 2000). Using the polar representation $z(\mathbf{r})=Z(\mathbf{r}) e^{2 i p \phi(\mathbf{r})}$, the eigenmode can be rewritten as $B_{p}^{+} Z\left(\mathbf{r}_{j}\right) \cos \left(2 p\left[\phi-\phi\left(\mathbf{r}_{j}\right)\right]\right)$. Thus, the maximum (linear) response of the $j$ th hypercolumn occurs at the orientation $\phi\left(\mathbf{r}_{j}\right)+k \pi / p, k=0,1, \ldots p-1$ for $p>0$.

In terms of the orientation tuning properties observed in real neurons, the most relevant cases are $p=0$ and $p=1$. The former corresponds to a bulk instability within a hypercolumn in which the new steady state exhibits no orientation preference. We call such a response the Hubel-Wiesel mode since any orientation tuning must be extrinsic to V1, generated, for example, by local anisotropies of the geniculo-cortical map (Hubel \& Wiesel, 1962). A bulk instability will occur when the local inhibition is sufficiently weak. In the second case, each hypercolumn supports an activity profile consisting of a solitary peak centered about the angle $\phi\left(\mathbf{r}_{j}\right)$, that is, the population response is characterized by an orientation tuning curve. One mechanism that generates a $p>0$ mode is if the local connections comprise short-range excitation and longer-range inhibition, which would be the case if the inhibitory neurons are identified with basket cells $(I=B a)$ whose lateral axonal spread has a space constant of about $250 \mu \mathrm{m}$. However, it is also possible within a two-population model to generate orientation tuning in the presence of short-range inhibition, as would occur if the inhibitory neurons are identified with local interneurons $(I=M a)$ instead whose lateral axonal spread is about $20 \mu \mathrm{m}$ (see Figure 1). Example spectra $W_{n}^{+}$for both of these cases are plotted in Figure 4 for gaussian coefficients

$$
W_{l m}(n)=\sqrt{2 \pi} \xi_{l m} \alpha_{l m} \mathrm{e}^{-n^{2} \xi_{l m}^{2} / 2},
$$



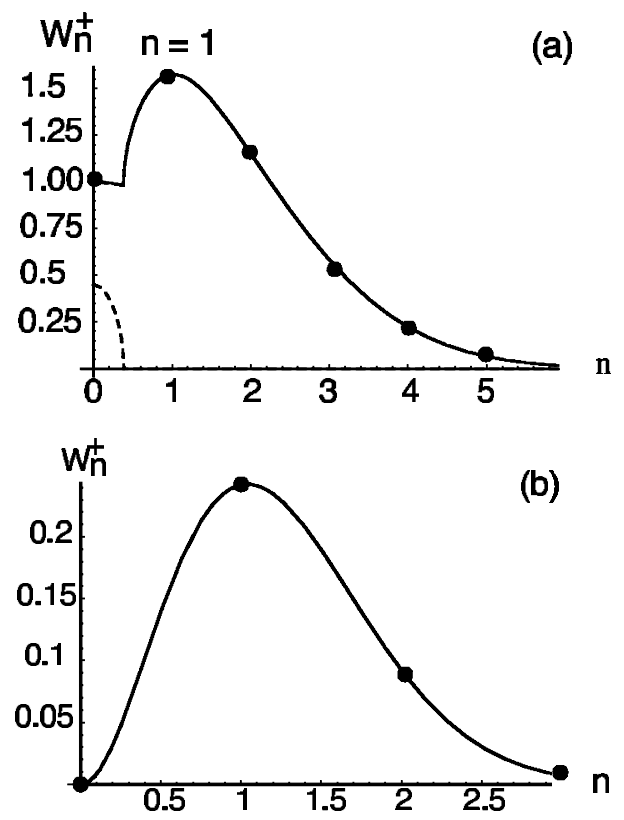

Figure 4: (a) Distribution of eigenvalues $W_{n}^{+}$as a function of $n$ in the case of long-range inhibition. Parameter values are $\xi_{E E}=\xi_{I E}=15^{\circ}, \xi_{I I}=\xi_{E I}=60^{\circ}$, $\alpha_{E E}=1, \alpha_{E I} \alpha_{I E}=0.3, \alpha_{I I}=0$. (b) Distribution of eigenvalues $W_{n}^{+}$as a function of $n$ in the case of short-range inhibition. Parameter values are $\xi_{E E}=35^{\circ}, \xi_{I E}=40^{\circ}$, $\xi_{I I}=\xi_{E I}=5^{\circ}, \alpha_{E E}=0.5, \alpha_{E I} \alpha_{I E}=0.5, \alpha_{I I}=1$. Solid line shows real part and dashed line shows the imaginary part.

where $\xi_{l m}$ determine the range of the axonal fields of the excitatory and inhibitory populations. For both examples, it can be seen that $\operatorname{Re}_{1}^{+}>\operatorname{Re} W_{n}^{+}$ for all $n \neq 1$ so that the $n=1$ mode becomes marginally stable first, leading to the spontaneous formation of sharp orientation tuning curves. (Note that if $\operatorname{Im} W_{1}^{+} \neq 0$ at the critical point, then the homogeneous resting state bifurcates to an orientation tuning curve whose peak spontaneously either rotates as a traveling wave or pulsates as a standing wave at a frequency determined by $\operatorname{Im} \lambda_{1}^{+}$(Ben-Yishai et al., 1997; Bressloff et al., 2000). We consider the time-periodic case in Section 3.3.

The location of the peak $\phi^{*}\left(\mathbf{r}_{j}\right)$ of the tuning curve at $\mathbf{r}_{j}$ is arbitrary in the presence of $\phi$-independent inputs $h_{l}\left(\mathbf{r}_{j}, \phi\right)=\bar{h}_{l}\left(\mathbf{r}_{j}\right)$. However, the inclusion of an additional small-amplitude input $\Delta h_{l}\left(\mathbf{r}_{j}, \phi\right) \sim \cos \left[2\left(\phi-\Phi_{j}\right)\right]$ breaks the rotational invariance of the system and locks the location of the tuning curve to the orientation corresponding to the peak of the stimulus, that is, $\phi^{*}\left(\mathbf{r}_{j}\right)=\Phi_{j}$. This is illustrated in Figure 5, where the input and output 


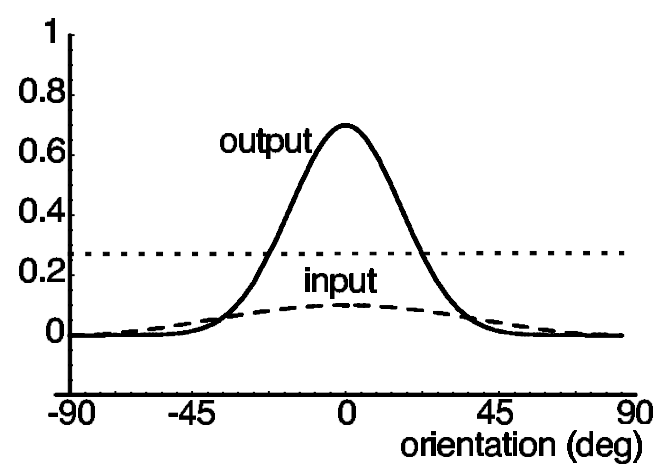

Figure 5: Sharp orientation tuning curve in a single hypercolumn. Local recurrent excitation and inhibition amplifies a weakly modulated input from the LGN. Dotted line is the baseline output without orientation tuning.

of the excitatory population of a single hypercolumn are shown (see also Section 4.1). Thus, the local intracortical connections within a hypercolumn serve to amplify a weakly oriented input signal from the LGN (Somers et al., 1995; Ben-Yishai et al., 1997).

The proportion of thalamocortical versus intracortical input in vivo and the tuning of the thalamocortical input are matters of ongoing debate. It is still not known for certain whether orientation selectivity is introduced in a feedforward manner to subsequent layers or arises from the intrinsic recurrent architecture of the upper layers of layer 4 or higher, or from some combination of these mechanisms. Recent work appears to confirm the original proposal of Hubel and Wiesel (1962) that geniculate input is sharply tuned in the cat (Ferster, Chung, \& Wheat, 1997). On the other hand, a considerable body of work indicates that intracortical processes, including recurrent excitation and intracortical inhibition, are important in determining orientation selectivity (Blakemore \& Tobin, 1972; Sillito, 1975; Douglas et al., 1995; Somers et al., 1995). In primates such as the macaque, most layer IV C cells receiving direct thalamocortical input are unoriented, and thus the introduction of orientation asymmetry is clearly a cortical process. If orientation information is to be represented cortically and kept "on-line" for any period of time, then specific intracortical circuitry not too dissimilar from the tuning circuitry of the primate (e.g., local recurrent excitation and inhibition) is necessary to stably represent the sharply tuned input. The work of Phleger and Bonds (1995) demonstrating a loss of stable orientation tuning in cats with blocking of intracortical inhibition supports this view.

3.2 Cubic Amplitude Equation: Stationary Case. So far we have established that in the absence of lateral connections, each hypercolumn (labeled 
by cortical position $\mathbf{r}_{j}$ ) can exhibit sharp orientation tuning when in a sufficiently excited state. The peak of the tuning curve is fixed by inputs from the LGN signaling the presence of a local oriented bar in the classical receptive field (CRF) of the given hypercolumn. We wish to investigate how such activity is further modulated by stimuli outside the CRF due to the presence of anisotropic lateral connections between hypercolumns leading to contextual effects.

Our approach will be to exploit the fact that the lateral connections are weak relative to local circuit connections and to use bifurcation analysis to derive dynamical equations for the amplitudes of the excited modes close to the bifurcation point. These equations then allow us to explore the effects of local and lateral inputs on sharp orientation tuning. For simplicity, we assume that each hypercolumn can be in one of two states of activation distinguished by the index $\chi\left(\mathbf{r}_{i}\right)=0,1$, where $\mathbf{r}_{i}$ is the cortical position of the $i$ th hypercolumn. If $\chi\left(\mathbf{r}_{i}\right)=0$, then the neurons within the hypercolumn are well below threshold so that there is only spontaneous activity, $\sigma \approx$ 0 . On the other hand, if $\chi\left(\mathbf{r}_{i}\right)=1$, then the hypercolumn exhibits sharp orientation tuning with mean levels of output activity $\sigma\left(\bar{a}_{l}\left(\mathbf{r}_{i}\right)\right)$ where $\bar{a}_{l}\left(\mathbf{r}_{i}\right)$ is a fixed-point solution of equations 3.4 and 3.5 close to the bifurcation point. Any hypercolumn in the active state is taken to have the same mean output activity. That is, if we denote the set of active hypercolumns by $\mathcal{J}=\left\{i \in 1, \ldots, N ; \chi\left(\mathbf{r}_{i}\right)=1\right\}$, then $\bar{a}_{l}\left(\mathbf{r}_{i}\right)=\bar{a}_{l}$ for all $i \in \mathcal{J}$, and we set $\sigma\left(\bar{a}_{l}\right)=\bar{\sigma}$.

First, perform a Taylor expansion of equation 2.4 with respect to $b_{l}\left(\mathbf{r}_{i}, \phi, t\right)$ $=a_{l}\left(\mathbf{r}_{i}, \phi, t\right)-\bar{a}_{l}, i \in \mathcal{J}$,

$$
\begin{aligned}
\frac{\partial b_{l}}{\partial t}= & -b_{l}+\sum_{m=E, I} w_{l m} *\left[\mu b_{m}+\gamma_{m} b_{m}^{2}+\gamma_{m}^{\prime} b_{m}^{3}+\ldots\right]+\Delta h_{l} \\
& +\epsilon \beta_{l} \hat{w} \circ\left(\left[\bar{\sigma}_{E}+\mu b_{E}+\ldots\right] \chi\right)
\end{aligned}
$$

where $\Delta h_{l}=h_{l}-\bar{h}_{l}$ and $\mu=\sigma_{l}^{\prime}\left(\bar{a}_{l}\right), \gamma_{l}=\sigma^{\prime \prime}\left(\bar{a}_{l}\right) / 2, \gamma_{l}^{\prime}=\sigma^{\prime \prime \prime}\left(\bar{a}_{l}\right) / 6$. The convolution operation $*$ is defined by equation 3.3 and

$$
[\hat{w} \circ f]\left(\mathbf{r}_{i}, \phi\right)=\sum_{j \in \mathcal{J}, j \neq i} \hat{w}\left(r_{i j}\right) \int_{-\pi / 2}^{\pi / 2} p_{0}\left(\phi^{\prime}-\theta_{i j}\right) p_{1}\left(\phi-\phi^{\prime}\right) f\left(\mathbf{r}_{j}, \phi^{\prime}\right) \frac{d \phi^{\prime}}{2 \pi^{\prime}}
$$

for an arbitrary function $f(\mathbf{r}, \phi)$, and $\hat{w}$ given by equation 2.5 . Suppose that the system is $\epsilon$-close to the point of marginal stability of the homogeneous fixed point associated with excitation of the modes $\mathrm{e}^{ \pm 2 i \phi}$. That is, take $\mu=$ $\mu_{c}+\epsilon \Delta u$ where $\mu_{c}=1 / W_{1}^{+}$(see equation 3.11). Substitute into equation 3.16 
the perturbation expansion,

$$
b_{m}=\epsilon^{1 / 2} b_{m}^{(1)}+\epsilon b_{m}^{(2)}+\epsilon^{3 / 2} b_{m}^{(3)}+\cdots
$$

Finally, introduce a slow timescale $\tau=\epsilon t$ and collect terms with equal powers of $\epsilon$. This leads to a hierarchy of equations of the form (up to $\mathcal{O}\left(\epsilon^{3 / 2}\right)$ ):

$$
\begin{aligned}
{\left[\mathcal{L} \mathbf{b}^{(1)}\right]_{l}=} & 0 \\
{\left[\mathcal{L} \mathbf{b}^{(2)}\right]_{l}=} & v_{l}^{(2)} \\
\equiv & \sum_{m=E, I} \gamma_{m} w_{l m} *\left[b_{m}^{(1)}\right]^{2}+\beta_{l} \bar{\sigma}_{E} \hat{w} \circ \chi \\
{\left[\mathcal{L} \mathbf{b}^{(3)}\right]_{l}=} & v_{l}^{(3)} \\
\equiv & -\frac{\partial b_{l}^{(1)}}{\partial \tau}+\sum_{m=E, I} w_{l m} *\left[\Delta \mu b_{m}^{(1)}+\gamma_{m}^{\prime}\left[b_{m}^{(1)}\right]^{3}+2 \gamma_{m} b_{m}^{(1)} b_{m}^{(2)}\right] \\
& +\Delta h_{l}+\mu_{c} \beta_{l} \hat{w} \circ\left(b_{E}^{(1)} \chi\right)
\end{aligned}
$$

with the linear operator $\mathcal{L}$ defined according to

$$
[\mathcal{L} \mathbf{b}]_{l}=b_{l}-\mu_{c} \sum_{m=E, I} w_{l m} * b_{m} .
$$

We have also assumed that the modulatory external input is $\mathcal{O}\left(\epsilon^{3 / 2}\right)$ and rescaled $\Delta h_{l} \rightarrow \epsilon^{3 / 2} \Delta h_{l}$.

Recall that each active hypercolumn is assumed to exhibit sharp orientation tuning, so that the local connections are such that the first equation in the hierarchy, equation 3.19, has solutions of the form

$$
\mathbf{b}^{(1)}\left(\mathbf{r}_{i}, \phi, \tau\right)=\left(z\left(\mathbf{r}_{i}, \tau\right) \mathrm{e}^{2 i \phi}+\bar{z}\left(\mathbf{r}_{i}, \tau\right) \mathrm{e}^{-2 i \phi}\right) \mathbf{B}
$$

for all $i \in \mathcal{J}$, with $\mathbf{B} \equiv \mathbf{B}_{1}^{+}$defined in equation 3.14 and $\bar{z}$ denotes the complex conjugate of $z$. We obtain a dynamical equation for the complex amplitude $z\left(\mathbf{r}_{i}, \tau\right)$ by deriving solvability conditions for the higher-order equations. We proceed by taking the inner product of equations 3.20 and 3.21 with the dual eigenmode $\widetilde{\mathbf{b}}(\phi)=\mathrm{e}^{2 i \phi} \widetilde{\mathbf{B}}$ where

$$
\widetilde{\mathbf{B}}=\left(\begin{array}{c}
W_{I E}(1) \\
-\frac{1}{2}\left[W_{E E}(1)+W_{I I}(1)-\Sigma(1)\right]
\end{array}\right)
$$

so that

$$
\left[\mathcal{L}^{T} \widetilde{\mathbf{b}}\right]_{l} \equiv \widetilde{b}_{l}-\mu_{c} \sum_{m=E, I} w_{m l} * \widetilde{b}_{m}=0 .
$$


The inner product of any two vector-valued functions of $\phi$ is defined as

$$
\langle\mathbf{u} \mid \mathbf{v}\rangle=\int_{0}^{\pi}\left[\bar{u}_{E}(\phi) v_{E}(\phi)+\bar{u}_{I}(\phi) v_{I}(\phi)\right] \frac{d \phi}{\pi} .
$$

With respect to this inner product, the linear operator $\mathcal{L}$ satisfies $\langle\widetilde{\mathbf{b}} \mid \mathcal{L} \mathbf{b}\rangle=$ $\left\langle\mathcal{L}^{T} \widetilde{\mathbf{b}} \mid \mathbf{b}\right\rangle=0$ for any $\mathbf{b}$. Since $\mathcal{L} \mathbf{b}^{(p)}=\mathbf{v}^{(p)}$, we obtain a hierarchy of solvability conditions $\left\langle\tilde{\mathbf{b}} \mid \mathbf{v}^{(p)}\right\rangle=0$ for $p=2,3, \ldots$

It can be shown from equations $3.17,3.20$, and 3.23 that the first solvability condition requires that

$$
\beta_{l} \bar{\sigma}_{E} P_{0}^{(1)} P_{1}^{(1)} \sum_{j \in \mathcal{J}} \hat{w}\left(r_{i j}\right) \mathrm{e}^{-2 i \theta_{i j}} \leq \mathcal{O}\left(\epsilon^{1 / 2}\right)
$$

where

$$
P_{k}^{(n)}=\left[\int_{-\pi / 2}^{\pi / 2} \mathrm{e}^{-2 n i \phi} p_{k}(\phi) \frac{d \phi}{\pi}\right] .
$$

This is analogous to the so-called adaptation condition in the bifurcation theory of weakly connected neurons (Hoppensteadt \& Izhikevich, 1997). If it is violated, then at this level of approximation, the interactions between hypercolumns become trivial. There are two ways in which equation 3.26 can be satisfied: the configuration of surrounding hypercolumns is such that $\sum_{j} \hat{w}\left(r_{i j}\right) \mathrm{e}^{-2 i \theta_{i j}} \leq \mathcal{O}\left(\epsilon^{1 / 2}\right)$ or the mean firing rate $\bar{\sigma}_{E} \leq \mathcal{O}\left(\epsilon^{1 / 2}\right)$. Here we assume that $\bar{\sigma}_{E}=\mathcal{O}\left(\epsilon^{1 / 2}\right)$.

The solvability condition $\left\langle\widetilde{\mathbf{b}} \mid \mathbf{v}^{(3)}\right\rangle=0$ generates a cubic amplitude equation for $z\left(\mathbf{r}_{i}, \tau\right)$. As a further simplification, we set $\gamma_{m}=0$, since this does not alter the basic structure of the amplitude equation. (Note, however, that the coefficients of the amplitude equation are $\gamma_{m}$ dependent, and hence the stability properties of a pattern may change if $\gamma_{m} \neq 0$.) Using equations 3.17, 3.21 , and 3.23, we then find that (after rescaling $\tau$ )

$$
\begin{aligned}
\frac{\partial z\left(\mathbf{r}_{i}, \tau\right)}{\partial \tau}= & z\left(\mathbf{r}_{i}, \tau\right)\left(\Delta \mu-A\left|z\left(\mathbf{r}_{i}, \tau\right)\right|^{2}\right)+f\left(\mathbf{r}_{i}\right)+\beta \sum_{j \in \mathcal{J}} \hat{w}\left(r_{i j}\right) \\
& \times\left[z\left(\mathbf{r}_{j}, \tau\right)+\bar{z}\left(\mathbf{r}_{j}, \tau\right) P_{0}^{(2)} \mathrm{e}^{-4 i \theta_{i j}}+\sigma P_{0}^{(1)} \mathrm{e}^{-2 i \theta_{i j}}\right],
\end{aligned}
$$

for all $i \in \mathcal{J}$ where $\sigma=\bar{\sigma}_{E} / \sqrt{\epsilon} \mu_{c} B_{E}$,

$$
\begin{aligned}
\beta & =P_{1}^{(1)} \sum_{l=E, I} D_{l} \beta_{l} \\
f\left(\mathbf{r}_{i}\right) & =\mu_{c} \sum_{l=E, I} \widetilde{B}_{l} \int_{0}^{\pi} \mathrm{e}^{-2 i \phi} \Delta h_{l}\left(\mathbf{r}_{i}, \phi\right) \frac{d \phi}{\pi}
\end{aligned}
$$


and

$$
D_{l}=\frac{\mu_{c}^{2} B_{E}}{\widetilde{\mathbf{B}}^{T} \mathbf{B}} \widetilde{B}_{l}, \quad A=-\frac{3}{\widetilde{\mathbf{B}}^{T} \mathbf{B}} \sum_{l=E, I} \widetilde{B}_{l} \gamma_{l}^{\prime} B_{l}^{3} .
$$

Equation 3.28 is our reduced model of weakly interacting hypercolumns. It describes the effects of anisotropic lateral connections and modulatory inputs from the LGN on the dynamics of the (complex) amplitude $z\left(\mathbf{r}_{i}, \tau\right)$. The latter determines the response properties of the orientation tuning curve associated with the hypercolumn at cortical position $\mathbf{r}_{i}$. The coupling parameter $\beta$ is a linear combination of the relative strengths of the lateral connections' innervating excitatory neurons and those innervating inhibitory neurons, with $D_{E}, D_{I}$ determined by the local weight distribution. Since $D_{E}>0$ and $D_{I}<0$, we see that the effective interactions between hypercolumns have both an excitatory and an inhibitory component. (The factor $P_{1}^{(1)}$ appearing in equation 3.29, which arises from the spread in lateral connections with respect to orientation-see Figure $3 b$ - generates a positive rescaling of the lateral interactions and can be absorbed into the parameters $D_{E}$ and $D_{I}$.) Note that in the case of isotropic lateral connections, $P_{k}^{(n)}=0$ for all $n>1$ so that $z$ decouples from $\bar{z}$ in the amplitude equation 3.28 .

3.3 Cubic Amplitude Equation: Oscillatory Case. In our derivation of the amplitude equation, 3.28 , we assumed that the local cortical circuit generates a stationary orientation tuning curve. However, as shown in Section 3.1, it is possible for a time-periodic tuning curve to occur when $\operatorname{ImW}_{1}^{+} \neq 0$. Taylor expanding 2.4 as before leads to the hierarchy of equations, 3.19 through 3.21, except that the linear operator $\mathcal{L} \rightarrow \mathcal{L}_{t}=\mathcal{L}+\partial / \partial t$. The lowest-order solution, equation 3.23, now takes the form

$$
\mathbf{b}^{(1)}\left(\mathbf{r}_{i}, \phi, t, \tau\right)=\left[z_{L}\left(\mathbf{r}_{i}, \tau\right) \mathrm{e}^{i\left(\Omega_{0} t-2 \phi\right)}+z_{R}\left(\mathbf{r}_{i}, \tau\right) \mathrm{e}^{i\left(\Omega_{0} t+2 \phi\right)}\right] \mathbf{B}+\text { c.c. },
$$

where $z_{L}$ and $z_{R}$ represent the complex amplitudes for anticlockwise $(L)$ and clockwise $(R)$ rotating waves (around the ring of a single hypercolumn), and

$$
\Omega_{0}=\mu_{c} \sqrt{4 W_{E I}(1) W_{I E}(1)-\left[W_{E E}(1)+W_{I I}(1)\right]^{2}} .
$$

Introduce the generalized inner product,

$\langle\mathbf{u} \mid \mathbf{v}\rangle=\lim _{T \rightarrow \infty} \frac{1}{T} \int_{-T / 2}^{T / 2} \int_{0}^{\pi}\left[\bar{u}_{E}(\phi, t) v_{E}(\phi, t)+\bar{u}_{I}(\phi, t) v_{I}(\phi, t)\right] \frac{d \phi}{\pi} d t$,

and the dual vectors $\widetilde{\mathbf{b}}_{L}=\widetilde{\mathbf{B}} \mathrm{e}^{i\left(\Omega_{0} t-2 \phi\right)}, \widetilde{\mathbf{b}}_{R}=\widetilde{\mathbf{B}}^{i\left(\Omega_{0} t+2 \phi\right)}$. Using the fact that $\left\langle\widetilde{\mathbf{b}}_{L} \mid \mathcal{L}_{t} b\right\rangle=\left\langle\widetilde{\mathbf{b}}_{R} \mid \mathcal{L}_{t} \mathbf{b}\right\rangle=0$ for arbitrary $\mathbf{b}$, we obtain the pair of solvability conditions $\left\langle\tilde{\mathbf{b}}_{L} \mid \mathbf{v}^{(p)}\right\rangle=\left\langle\tilde{\mathbf{b}}_{R} \mid \mathbf{v}^{(p)}\right\rangle=0$ for each $p \geq 2$. 
The $p=2$ solvability conditions are identically satisfied. The $p=3$ solvability conditions then generate cubic amplitude equations for $z_{L}, z_{R}$ of the form

$$
\begin{aligned}
\frac{\partial z_{L}\left(\mathbf{r}_{i}, \tau\right)}{\partial \tau}= & \left(1+i \Omega_{0}\right) z_{L}\left(\mathbf{r}_{i}, \tau\right)\left[\Delta \mu-A\left|z_{L}\left(\mathbf{r}_{i}, \tau\right)\right|^{2}-2 A\left|z_{R}\left(\mathbf{r}_{i}, \tau\right)\right|^{2}\right] \\
& +f_{-}\left(\mathbf{r}_{i}\right)+\beta \sum_{j \in \mathcal{J}} \hat{w}\left(r_{i j}\right)\left[z_{L}\left(\mathbf{r}_{j}, \tau\right)+z_{R}\left(\mathbf{r}_{j}, \tau\right) P_{0}^{(2)} \mathrm{e}^{4 i \theta_{i j}}\right]
\end{aligned}
$$

and

$$
\begin{aligned}
\frac{\partial z_{R}\left(\mathbf{r}_{i}, \tau\right)}{\partial \tau}= & \left(1+i \Omega_{0}\right) z_{R}\left(\mathbf{r}_{i}, \tau\right)\left[\Delta \mu-A\left|z_{R}\left(\mathbf{r}_{i}, \tau\right)\right|^{2}-2 A\left|z_{L}\left(\mathbf{r}_{i}, \tau\right)\right|^{2}\right] \\
& +f_{+}\left(\mathbf{r}_{i}\right)+\beta \sum_{j \in \mathcal{J}} \hat{w}\left(r_{i j}\right)\left[z_{R}\left(\mathbf{r}_{j}, \tau\right)+z_{L}\left(\mathbf{r}_{j}, \tau\right) P_{0}^{(2)} \mathrm{e}^{-4 i \theta_{i j}}\right],
\end{aligned}
$$

where

$$
f_{ \pm}\left(\mathbf{r}_{i}\right)=\lim _{T \rightarrow \infty} \frac{\mu_{c}}{T} \int_{-T / 2}^{T / 2} \int_{0}^{\pi} \mathrm{e}^{-i\left(\Omega_{0} t \pm 2 \phi\right)} \sum_{l=E, I} \widetilde{B}_{l} \Delta h_{l}\left(\mathbf{r}_{i}, \phi, t\right) \frac{d \phi}{\pi} d t
$$

It can be seen that the amplitudes couple only to time-dependent inputs from the LGN.

\section{Contextual Effects}

In the previous section, we used perturbation techniques to reduce the original infinite-dimensional system of Wilson-Cowan equations (2.4) to a corresponding finite-dimensional system of amplitude equations (3.28). This is illustrated in Figure 6. The complex amplitude $z\left(\mathbf{r}_{i}\right)=Z_{i} \mathrm{e}^{-2 i \phi_{i}}$ determines the linear response function $R_{i}(\phi)$ of the $i$ th hypercolumn according to

$$
R_{i}(\phi)=z\left(\mathbf{r}_{i}\right) \mathrm{e}^{2 i \phi}+\bar{z}_{i}\left(\mathbf{r}_{i}\right) \mathrm{e}^{-2 i \phi}=2 Z_{i} \cos \left(2\left[\phi-\phi_{i}\right]\right),
$$

and this in turn determines the population tuning curves of the excitatory and inhibitory populations. In this section, we use the amplitude equation, 3.28 , to investigate (at least qualitatively) how contextual stimuli falling outside the classical receptive field of a hypercolumn modify their response to stimuli within the classical receptive field; such contextual effects are mediated by the lateral interactions between hypercolumns.

4.1 A Single Driven Hypercolumn. We begin by determining the response of a single hypercolumn to an LGN input in the absence of lateral interactions $(\beta=0)$ and under the assumption that the orientation tuning 


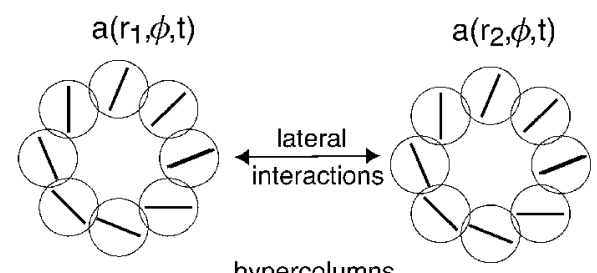

hypercolumns
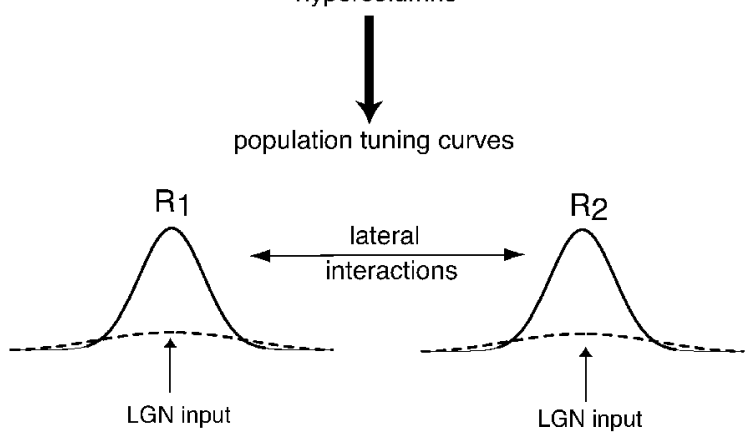

Figure 6: Reduction from a dynamical model of interacting hypercolumns to a dynamical model of interacting population tuning curves.

curves and external stimuli are stationary. Let the input from the LGN to the $i$ th active hypercolumn be of the form

$$
\Delta h_{l}\left(\mathbf{r}_{i}, \phi\right)=g_{l} C_{i} \cos \left(2\left[\phi-\Phi_{i}\right]\right)
$$

where $C_{i}, C_{i}>0$, is proportional to the contrast of the center stimulus, $\Phi_{i}$ is its orientation, and $g_{l} \geq 0$ determines the relative strengths of the feedforward input to the local excitatory and inhibitory populations. Such a stimulus represents an oriented edge or bar in the aggregate receptive field of the hypercolumn. It then follows from equation 3.30 that

$$
f\left(\mathbf{r}_{i}\right)=C_{i} \mathrm{e}^{-2 i \Phi_{i}}
$$

where we have set $\mu_{c} \sum_{l=E, I} \tilde{B}_{l} g_{l}=1$ for convenience. This term is positive if $g_{E} \gg g_{I}$. Setting $\beta=0$ in equation 3.28 and writing $z\left(\mathbf{r}_{i}\right)=Z_{i} \mathrm{e}^{-2 i \phi_{i}}$, with $Z_{i}$ real, we obtain the following pair of equations:

$$
\begin{aligned}
& \frac{\mathrm{d} Z_{i}}{\mathrm{~d} \tau}=Z_{i}\left(\Delta \mu-A Z_{i}^{2}\right)+C_{i} \cos \left(2\left[\phi_{i}-\Phi_{i}\right]\right) \\
& \frac{\mathrm{d} \phi_{i}}{\mathrm{~d} \tau}=-\frac{C_{i}}{2 Z_{i}} \sin \left(2\left[\phi_{i}-\Phi_{i}\right]\right) .
\end{aligned}
$$

Assuming that the coefficients $\Delta \mu>0, A>0$, these have a stable fixedpoint solution $\left(\phi_{i}^{*}, Z_{i}^{*}\right)$ with $\phi_{i}^{*}=\Phi_{i}$ (independent of the contrast), and $Z_{i}^{*}$ 
is the positive root of the cubic $Z_{i}\left(\Delta u-A Z_{i}^{2}\right)+C_{i}=0$. Thus, the hypercolumn encodes the orientation $\Phi_{i}$ of a local bar in its aggregate receptive field by locking the peak of its tuning curve to $\Phi_{i}$ (see Figure 5). The amplitude of the tuning curve is determined by $Z_{i}^{*}$, which is an increasing function of contrast. Note that in the absence of an external stimulus $\left(C_{i}=0\right)$, the tuning curve is marginally stable since $\phi_{i}^{*}$ is arbitrary. This means that the phase will be susceptible to noise-induced diffusion. Recall from Section 3 that the amplitude of the LGN input was assumed to be $\mathcal{O}\left(\epsilon^{3 / 2}\right)$, whereas the amplitude of the response is $\mathcal{O}\left(\epsilon^{1 / 2}\right)$. Since $\epsilon \ll 1$, the intrinsic circuitry of the hypercolumn amplifies the LGN input.

Now consider the oscillatory case. In order that the amplitudes $z_{L}$ and $z_{R}$ of equations 3.35 and 3.36 couple to an LGN input, the latter must be time dependent with a Fourier component at the natural frequency of oscillation $\Omega_{0}$ of the hypercolumn. It is certainly reasonable to assume that external stimuli have a time-dependent part. For example, neurophysiological experiments often use flashing or drifting oriented gratings as external stimuli. (These are chosen to compensate for the spike frequency adaptation of neurons in the cortex.) Time-dependent signals might also be generated in the LGN. Suppose that $C_{i}$ is the contrast of the relevant temporal frequency component and $\Phi_{i}$ is the orientation of the external stimulus. Neglecting lateral inputs, the amplitude equations 3.35 and 3.36 become

$$
\begin{aligned}
\frac{\partial z_{L}\left(\mathbf{r}_{i}, \tau\right)}{\partial \tau}= & \left(1+i \Omega_{0}\right) z_{L}\left(\mathbf{r}_{i}, \tau\right) \\
& \times\left[\Delta \mu-A\left|z_{L}\left(\mathbf{r}_{i}, \tau\right)\right|^{2}-2 A\left|z_{R}\left(\mathbf{r}_{i}, \tau\right)\right|^{2}\right]+C_{i} \mathrm{e}^{-2 i \Phi_{i}}
\end{aligned}
$$

and

$$
\begin{aligned}
\frac{\partial z_{R}\left(\mathbf{r}_{i}, \tau\right)}{\partial \tau}= & \left(1+i \Omega_{0}\right) z_{R}\left(\mathbf{r}_{i}, \tau\right) \\
& \times\left[\Delta \mu-A\left|z_{R}\left(\mathbf{r}_{i}, \tau\right)\right|^{2}-2 A\left|z_{L}\left(\mathbf{r}_{i}, \tau\right)\right|^{2}\right]+C_{i} \mathrm{e}^{2 i \Phi_{i}} .
\end{aligned}
$$

If $C_{i}=0, \Delta \mu>0$ and $A>0$, there are three types of fixed-point solution: (1) anticlockwise rotating waves $\left|z_{L}\right|^{2}=\Delta \mu / A, z_{R}=0$, (2) clockwise rotating waves $\left|z_{R}\right|^{2}=\Delta \mu / A, z_{L}=0$, and (3) standing waves $\left|z_{R}\right|^{2}=\left|z_{L}\right|^{2}=\Delta \mu / 3 A$. The standing waves are unstable, and the traveling waves are marginally stable due to the existence of arbitrary phases (Kath, 1981; Aronson, Ermentrout, \& Kopell, 1990). (Note, however, that it is possible to obtain stable standing waves when $\gamma_{m} \neq 0$ in equation 3.21.) If $C_{i}>0$, the traveling wave solutions no longer exist, but standing wave solutions do, of the form $z_{L}=Z_{i} \mathrm{e}^{-2 i \Phi_{i}} \mathrm{e}^{i \psi}, z_{R}=Z_{i} \mathrm{e}^{2 i \Phi_{i}} \mathrm{e}^{i \psi}$ with $\tan \psi=-\Omega_{0}$ and $Z_{i}$ a positive root of the cubic

$$
Z_{i}\left[\Delta \mu-3 A Z_{i}^{2}\right]+C_{i} \cos (\psi)=0 .
$$


Equation 3.32 then implies that the tuning curves are of the form

$$
4 Z_{i} \cos \left(\Omega_{0} t+\psi\right) \cos \left(2\left[\phi-\Phi_{i}\right]\right)
$$

It can be shown that these solutions are unstable for sufficiently low contrasts but stable for high contrasts.

The existence of a stable, time-periodic tuning curve in the case of a single isolated hypercolumn means that it effectively acts as a single giant oscillator. One could then investigate, for example, the synchronization properties of a network of hypercolumns coupled via anisotropic lateral interactions. However, it is not clear how to interpret these oscillations biologically. One possibility is that they correspond to the $40 \mathrm{~Hz} \gamma$ frequency oscillations that are thought to play a role in the synchronization of cell assemblies (Gray, Konig, Engel, \& Singer, 1989; Singer \& Gray, 1995). One difficulty with this interpretation is that the $\gamma$ oscillations appear in the spikes of individual neurons, and thus the mechanism for generating them is likely to be washed out in any mean-field theory analysis used to derive the rate models considered in this article. On the other hand, recent work on integrate-and-fire networks has shown that time-dependent firing rates can be viewed as modulations of single neuron spikes (Bressloff et al., 2000). Given the difficulties concerning the significance of oscillatory tuning curves, we assume that the normal operating regime of each hypercolumn is to generate stationary tuning curves in response to slowly changing LGN inputs.

4.2 Center-Surround Interactions. Consider the following experimental situation (Blakemore \& Tobin, 1972; Li \& Li, 1994; Sillito et al., 1995): a particular hypercolumn designated the "center" is stimulated with a grating at orientation $\Phi_{c}$, while outside the receptive area of this hypercolumn-in the "surround"- - there is a grating at some uniform orientation $\Phi_{s}$ as shown in Figure 7a. In order to analyze this problem, we introduce a mean-field approximation. That is, the active region of cortex responding to the centersuround stimulus of Figure $7 \mathrm{a}$ is taken to have the configuration shown in Figure $7 \mathrm{~b}$. This consists of a center hypercolumn at $\mathbf{r}_{c}=0$ interacting with a ring of $N$ identical surround hypercolumns at relative positions $\mathbf{r}_{j}$, with $\left|\mathbf{r}_{j}\right|=R, j=1, \ldots, N$. Note that the total input from surround to center is strong relative to that from center to surround. Therefore, as a further approximation, we can neglect the latter and treat the system as a single hypercolumn receiving a mixture of inputs from the LGN and lateral inputs from the surround (see Figure 7c).

Suppose that the surround hypercolumns are sharply tuned to the stimulus orientation $\Phi_{s}$, that is, they have steady-state amplitudes of the form $z\left(\mathbf{r}_{j}\right)=Z_{s} \mathrm{e}^{-2 i \Phi_{s}}$, where $Z_{s}$ is positive and real. It follows from equation 3.28 that the complex amplitude $z_{\mathcal{c}}(\tau)$ characterizing the response of the center 


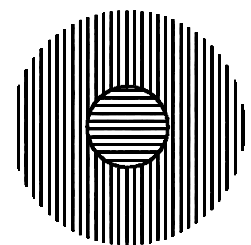

(a)

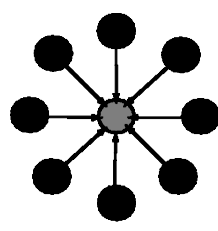

(b)

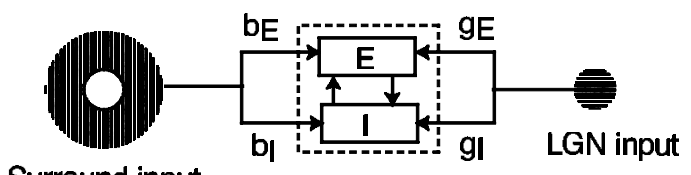

Surround input

(c)

Figure 7: (a) Circular center-surround stimulus configuration. (b) Cortical configuration of a center hypercolumn interacting with a ring of surround hypercolumns. (c) Effective single hypercolumn circuit in which there are direct LGN inputs from the center and lateral inputs from the surround. The relative strengths of the lateral inputs to the excitatory (E) and inhibitory (I) populations are determined by $\beta_{E}$ and $\beta_{I}$, respectively. The corresponding LGN input strengths are denoted by $g_{E}$ and $g_{I}$.

satisfies the equation

$$
\begin{aligned}
\frac{\mathrm{d} z_{c}(\tau)}{\mathrm{d} \tau}= & z_{\mathcal{c}}(\tau)\left(\Delta \mu-A\left|z_{c}(\tau)\right|^{2}\right) \\
& +\beta w_{s}\left(\mathrm{e}^{-2 i \Phi_{s}}+\Lambda \mathrm{e}^{2 i \Phi_{s}}+\tilde{\Lambda}\right)+C_{c} \mathrm{e}^{-2 i \Phi_{c}},
\end{aligned}
$$

where $w_{S}=N Z_{S} \hat{w}(R)>0, C_{c}$ is the contrast of the center stimulus and

$$
\Lambda=\frac{P_{0}^{(2)}}{N} \sum_{j=1}^{N} \mathrm{e}^{-4 i \theta_{j}}, \quad \tilde{\Lambda}=\frac{\sigma P_{0}^{(1)}}{N Z_{S}} \sum_{j=1}^{N} \mathrm{e}^{-2 i \theta_{j}},
$$

with $\theta_{j}$ the direction of the line from the center to the $j$ th surround hypercolumn (see Figure $7 \mathrm{~b}$ ). We now make the simplifying assumption that $\Lambda=\tilde{\Lambda}=0$ due to the rotational symmetry of the mean-field configuration. Writing $z_{c}=Z_{c} \mathrm{e}^{-2 i \phi_{c}}$, we then obtain the following pair of equations:

$$
\begin{aligned}
\frac{\mathrm{d} Z_{c}}{\mathrm{~d} \tau}= & Z_{c}\left(\Delta \mu-A Z_{c}^{2}\right)+C_{c} \cos \left(2\left[\phi_{c}-\Phi_{c}\right]\right) \\
& +\beta w_{s} \cos \left(2\left[\phi_{c}-\Phi_{s}\right]\right) \\
\frac{\mathrm{d} \phi_{c}}{\mathrm{~d} \tau}= & -\frac{C_{c}}{2 Z_{c}} \sin \left(2\left[\phi_{c}-\Phi_{c}\right]\right)-\frac{\beta w_{s}}{2 Z_{c}} \sin \left(2\left[\phi_{c}-\Phi_{s}\right]\right) .
\end{aligned}
$$



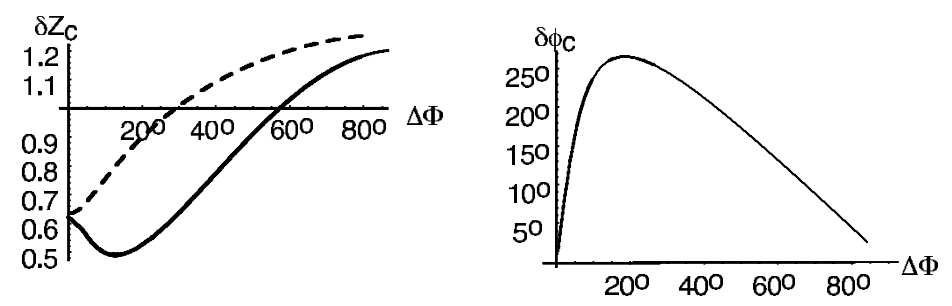

Figure 8: Response of center population as a function of the relative orientation of the surround stimulus $\Delta \Phi=\Phi_{s}-\Phi_{c}$. Parameters in amplitude equations 4.11 and 4.12 are $\Delta \mu=0.1,|\beta| \hat{w}_{s}=0.8, C_{c}=1$, and $A=1$. (a) Solid line: total response $\delta Z_{c}$ at preferred orientation as a fraction of the response in the absence of surround modulation. Dashed line: amplitude of maximum response $Z_{c}^{*}(\beta)$. (b) Plot of the shift in the peak of the orientation tuning curve $\delta \phi_{c}=\Phi_{c}-\phi_{c}^{*}(\beta)$.

Recall from equation 3.29 that the effective interaction parameter $\beta$ has both an excitatory and an inhibitory component. As noted previously, experimental data suggest that the effect of lateral inputs on the response of a hypercolumn is contrast sensitive, tending to be suppressive at high contrasts and facilitatory at low contrasts (Toth et al., 1996; Levitt \& Lund, 1997; Polat et al., 1998). In terms of the amplitude equation, 4.9, this implies that $\beta=\beta\left(C_{c}\right)$. For the moment, we assume that the contrast of the center stimulus is large enough so that $\beta<0$.

Let $\left(Z_{c}^{*}(\beta), \phi_{c}^{*}(\beta)\right)$ be the unique, stable, steady-state solution of equations 4.11 and 4.12 for a given $\beta$. We define two important quantities: the shift $\delta \phi_{c}$ in the peak of the tuning curve and the fractional change $\delta Z_{c}$ in the linear response at the preferred orientation $\Phi_{i}$,

$$
\delta \phi_{c}=\Phi_{c}-\phi_{c}^{*}(\beta), \quad \delta Z_{c}=\frac{Z_{c}^{*}(\beta) \cos \left(2 \delta \phi_{c}\right)}{Z_{c}^{*}(0)}
$$

If $\delta Z_{c}<1$, the effect of the lateral inputs is supressive, whereas if $\delta Z_{c}>$ 1 , then it is facilitatory. In Figure 8 we plot the resulting quantities $\delta Z_{c}$ and $\delta \phi_{c}$ as a function of $\Delta \Phi=\Phi_{s}-\Phi_{c}$. It can be seen that surround modulation changes from suppression to facilitation as the difference in the orientation of the surround and the center increases beyond around $60^{\circ}$. This is consistent with experimental results on center-surround suppression and facilitation at high contrasts (Blakemore \& Tobin, 1972; Li \& Li, 1994; Sillito et al., 1995). An important contribution to the asymmetry between suppression and facilitation arises from the shift in the peak of the effective tuning curve in the presence of the surround, as measured by $\delta \phi_{c}$. Since this is positive, there is an apparent increase in the difference between the center and surround tuning curve peaks, which is analogous to the direct tilt effect observed in psychophysical experi- 
ments (Wenderoth \& Johnstone, 1988), although the effect is much larger here.

We now give a simple argument for the switch from suppression to facilitation as the relative angle $\Delta \Phi=\Phi_{s}-\Phi_{c}$ is increased. If the surround is stimulated by a grating parallel to that of the center stimulus $\left(\Phi_{s}=\Phi_{c}\right)$, then $\phi_{c}^{*}=\Phi_{c}$ and equation 4.11 becomes

$$
\frac{\mathrm{d} Z_{c}}{\mathrm{~d} \tau}=Z_{c}\left(\Delta \mu-A Z_{c}^{2}\right)+C_{c}+\beta w_{s} .
$$

Similarly, in the case of an orthogonal surround $\left(\Phi_{s}=\Phi_{c}+90^{\circ}\right)$,

$$
\frac{\mathrm{d} Z_{c}}{\mathrm{~d} \tau}=Z_{c}\left(\Delta \mu-A Z_{c}^{2}\right)+C_{c}-\beta w_{s} .
$$

The steady-state amplitude $Z_{c}^{*}$ for $\beta=0$ is an increasing function of $C_{c}$ (see Section 4.1). Hence, if $\beta<0$, then $C_{c} \rightarrow C_{c}-\left|\beta w_{c}\right|$ for a collinear surround, resulting in a suppression of the center response, whereas $C_{c} \rightarrow C_{c}+\left|\beta w_{c}\right|$ for an orthogonal surround leading to facilitation.

The occurrence of a facilitatory response to an orthogonal surround stimulus has been observed experimentally by a number of groups (Blakemore \& Tobin, 1972; Sillito et al., 1995; Levitt \& Lund, 1997; Polat et al., 1998). At first sight, however, this conflicts with the consistent experimental finding that stimulating a hypercolumn with an orthogonal stimulus suppresses the response to the original stimulus. In particular, DeAngelis, Robson, Ohzawa, and Freeman (1992) show that cross-orientation suppression (with orthogonal gratings) originates within the receptive field of most cat neurons examined and is a consistent finding in both complex and simple cells. The degree of suppression depends linearly on the size of the orthogonal grating up to a critical dimension, which is smaller than the classical receptive field dimension. Such observations are compatible with the above findings. In the case of orthogonal inputs to the same hypercolumn, we have the simple linear summation $C_{c} \cos (2 \phi)+C_{c}^{\prime} \cos (2[\phi-\pi / 2])=\left(C_{c}-C_{c}^{\prime}\right) \cos (2 \phi)$, where $C_{c}>C_{c}^{\prime}>0$. Thus, the orthogonal input of amplitude $C_{c}^{\prime}$ reduces the amplitude of the original input and hence gives rise to a smaller response. On the other hand, the effect of an orthogonal surround stimulus is mediated by the lateral connections and (in the suppressive setting) is input primarily to the orthogonal inhibitory population, which can lead to disinhibition and consequently facilitation. Similar arguments were used by Mundel et al. (1997) and more recently by Dragoi and Sur (2000) in their numerical study of interacting hypercolumns.

The center response to surround stimulation depends significantly on the contrast of the center stimulation (Toth et al., 1996; Levitt \& Lund, 1997; Polat et al., 1998). For example, a fixed surround stimulus tends to facilitate responses to preferred orientation stimuli when the center contrast is low 


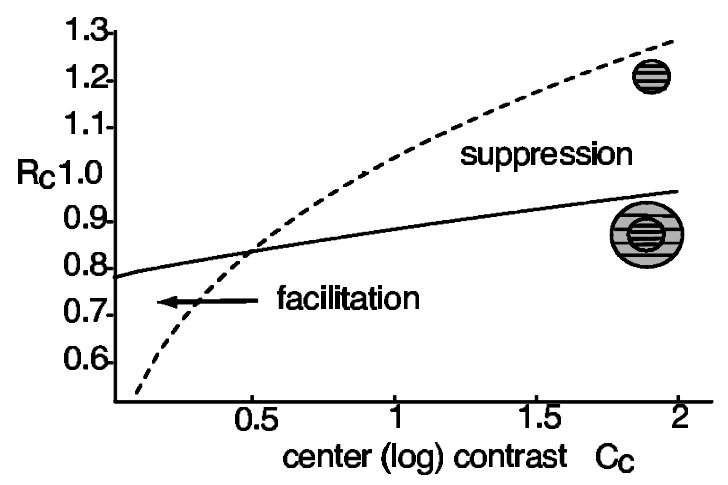

Figure 9: Variation in the response $R_{c}$ of a center hypercolumn at its preferred orientation as a function of $(\log )$ contrast $C_{c}$ for collinear high-contrast surround (solid curve) and no surround (dashed curve). Parameters in the amplitude equations 4.11 and 4.12 are $\Delta \mu=0.1, A=1$, and $\hat{w}_{s}=0.8$. The contrastdependent coupling is taken to be of the form $\beta=\left(0.5-C_{c}\right)$.

but suppresses responses when it is high. Both effects are strongest when the center and surround stimuli are at the same orientation. It has recently been shown that inclusion of some form of contrast-related asymmetry between local excitatory and inhibitory neurons is sufficient to account for the switch between low-contrast facilitation and high-contrast suppression (Somers et al., 1998).

One way to model the thresholding properties of the lateral interactions is to distinguish between the differing classes of inhibitory interneurons. For example, certain types of local interneuron are well placed to provide a source of feedforward inhibition from lateral connections, as illustrated in Figure 1. This feedforward inhibition will have its own threshold and gain that will be determined by the contrast of the LGN inputs, and there will thus be a contrast-dependent disynaptic inhibition arising from the lateral connections. We incorporate such an effect into our cortical model by including a contrast-dependent contribution to the lateral inputs of the excitatory population in equation 2.4: $\beta_{E} \rightarrow \beta_{E}-\beta^{*}$, where $\beta^{*}$ is contrast dependent. In the case of high contrasts, we expect $\beta^{*}$ to be sufficiently large so that the effective coupling $\beta<0$. This has been assumed in our analysis of high-contrast center-surround stimulation. Now suppose that we consider the response of the center in the presence of a high-contrast surround and varying center contrast. For the sake of illustration, suppose that $\beta^{*}$ varies linearly with the contrast. The response of the center at its preferred orientation, $R_{c}$, is plotted as a function of contrast in Figure 9, both with and without a collinear surround. It can be seen that there is facilitation at 
low contrasts and suppression at high contrasts, which is consistent with experimental data (Polat et al., 1998).

4.3 Anisotropy in Surround Suppression. So far we have considered a surround configuration in which the the anisotropic nature of the lateral connections is effectively averaged away. This is no longer the case if only a subregion of the surround is stimulated. For concreteness, suppose that only a single hypercolumn in the surround is active, and take its location relative to the center to be $\mathbf{r}=r(\cos \theta, \sin \theta)$ (see Figure $7 \mathrm{~b})$. Since the effects of feedback from the center hypercolumn to the surround cannot now be ignored, we have to solve the pair of equations

$$
\begin{aligned}
\frac{\mathrm{d} z_{\mathcal{c}}(\tau)}{\mathrm{d} \tau}= & z_{\mathcal{c}}(\tau)\left(\Delta \mu-A\left|z_{\mathcal{c}}(\tau)\right|^{2}\right)+C \mathrm{e}^{-2 i \Phi_{c}} \\
& +\beta \hat{w}(r)\left[z_{\mathcal{S}}+\bar{z}_{S} P_{0}^{(2)} \mathrm{e}^{-4 i \theta}+\sigma P_{0}^{(1)} \mathrm{e}^{-2 i \theta}\right] \\
\frac{\mathrm{d} z_{\mathcal{S}}(\tau)}{\mathrm{d} \tau}= & z_{\mathcal{S}}(\tau)\left(\Delta \mu-A\left|z_{\mathcal{S}}(\tau)\right|^{2}\right)+C \mathrm{e}^{-2 i \Phi_{s}} \\
& +\beta \hat{w}(r)\left[z_{\mathcal{c}}+\bar{z}_{\mathcal{C}} P_{0}^{(2)} \mathrm{e}^{-4 i \theta}+\sigma P_{0}^{(1)} \mathrm{e}^{-2 i \theta}\right],
\end{aligned}
$$

where for simplicity the contrast of the center and surround stimuli is taken to be equal. It is clear that if the lateral connections are isotropic, then $p_{0}(\phi)=$ 1 for all $\phi \in[-\pi / 2, \pi / 2)$, so that $P_{0}^{(2)}=0=P_{0}^{(1)}$, and the effective interaction between the center and surround is independent of their relative angular location $\theta$. However, if $P_{0}^{(1)}$ and $P_{0}^{(2)}$ are nonzero, then there will be some dependence on the relative angle $\theta$, which is a signature of the anisotropy of the lateral connections.

For the sake of illustration, suppose that $\Phi_{c}=\Phi_{s}=0$ (iso-oriented center and surround). Then, by symmetry, a fixed-point solution exists for which $z_{c}=z_{s}=Z \mathrm{e}^{-2 i \phi}$, where

$$
\begin{aligned}
& Z\left(\Delta \mu-A Z^{2}\right)+C \cos (2 \phi)+\beta \hat{w}(r) Z \\
& \quad \times\left[1+P_{0}^{(2)} \cos (4[\phi-\theta])+\sigma P_{0}^{(1)} \cos (2[\phi-\theta])\right]=0 \\
& C \sin (2 \phi)+\beta \hat{w}(r)\left[P_{0}^{(2)} \sin (4[\phi-\theta])+\sigma P_{0}^{(1)} \sin (2[\phi-\theta])\right]=0 .
\end{aligned}
$$

Some solutions of these equations are plotted in Figure 10 with $\delta Z_{c}=$ $Z(\beta) \cos (\phi) / Z(0)$. It can be seen that the degree of suppression of the center (at high-contrast) depends on both the relative angular position of the surround hypercolumn and the degree of spread in the lateral connections as characterized by the parameters $P_{0}^{(1)}$ and $P_{0}^{(2)}$. For certain parameter values 


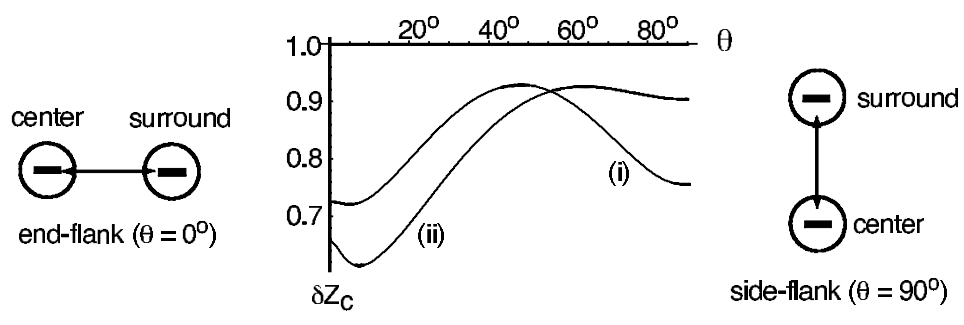

Figure 10: Variation in the degree of suppression of a center hypercolumn as a function of the relative angular position $\theta$ of a surround hypercolumn. Both center and surround are stimulated by a high-contrast horizontal bar such that $\theta=0^{\circ}$ corresponds to a collinear or end-flank configuration and $\theta=90^{\circ}$ corresponds to an orthogonal or side-flank configuration as shown. The two curves differ in the choice of spread parameters: (i) $\sigma P_{0}^{(1)}=0.1, P_{0}^{(2)}=0.6$ and (ii) $\sigma P_{0}^{(1)}=0.8, P_{0}^{(2)}=0.4$. Other parameters in equations 4.16 and 4.17 are $\mu=0.1$, $A=1, C=1.0$, and $|\beta| w(r)=0.5$.

(see curve ii in Figure 10), the suppressive effect of the surround hypercolumn is maximal when located close to the end-flank position and minimal when located around the side-flank position. However, it is also possible for the suppressive effect to be minimal at some oblique configuration (see curve i in Figure 10).

It is interesting that recent experimental data concerning the spatial organization of surrounds in primary visual cortex of the cat imply that in many cases, suppression originates from a localized region in the surround rather than being distributed uniformly in the region encircling the center's classical receptive field, that is, the surrounds are spatially asymmetric (Walker, Ohzawa, \& Freeman, 1999). The suppressive portion of the surround can arise at any location with a bias toward the ends of the center's classical receptive. Our analysis suggests that the effect of the surround depends on a subtle combination of the spread in the anisotropic lateral connections and the spatial organization of the surround.

\section{Discussion}

The mathematical model introduced here assumes that V1 dynamics is close to threshold, so that even weakly modulated signals from the LGN can trigger a tuned response. One way to achieve this is if there is a balance between intracortical excitation and inhibition. This and related ideas have been explored in a number of recent studies of the role of intracortical interactions on the observed properties of cortical neurons (Douglas et al., 1995; Carandini et al., 1997; Tsodyks \& Sejnowski, 1995; Chance \& Abbott, 2000; Wielaard, Shelley, McLaughlin, \& Shapley, 2001). 
5.1 Balanced Excitation and Inhibition. As discussed by Tsodyks and Sejnowski (1995), when a network with balanced excitation and inhibition is close to threshold, several properties obtain. (1) Fluctuations about the threshold are large, and remain so, even in the continuum limit. (2) The network switches discontinuously from threshold to a tuned excited state, indicating the existence of a subcritical bifurcation. (3) Critical slowing down occurs, that is, the network state changes more slowly from threshold to tuned the closer the stimulus is to threshold (see also Cowan \& Ermentrout, 1978) - given realistic neural parameters it takes about $50 \mathrm{msec}$ to reach the peak of the tuned state. However, (4) if the input orientation bias is switched instantaneously to a new value, the net switches to the new tuned state in less than $50 \mathrm{msec}$, particularly if the inhibition in the network is faster than the excitation. Finally, (5) the orientation-tuned response is contrast invariant. Most, if not all, of these properties are found in the mathematical model described above.

On a more technical level, we note that one consequence of having balanced excitation and inhibition is that to have any effect on the bifurcation process whereby new orientation tuned stable states emerge, external stimuli, for example, those from the LGN, which we labeled as $h_{l}\left(\mathbf{r}_{j}, \phi\right)$, must be of the form

$$
h_{l}\left(\mathbf{r}_{j}, \phi\right)=g_{l} C_{j}\left\{1-\epsilon^{3 / 2}+\epsilon^{3 / 2} \cos \left(2\left[\phi-\Phi_{j}\right]\right)\right\},
$$

where $g_{l}$ measures the relative strengths of LGN inputs to excitatory and inhibitory populations in $\mathrm{V} 1$, and $C_{j}$ is the stimulus contrast at the $j$ th hypercolumn. Thus, the nonoriented component $\left(1-\epsilon^{3 / 2}\right) g_{l} C_{j}$ is $0(1)$ and couples to the steady-state equations, whereas the orientation-tuned component $\epsilon^{3 / 2} g_{l} C_{j} \cos \left(2\left[\phi-\Phi_{j}\right]\right)$ is $0\left(\epsilon^{3 / 2}\right)$ and couples to the cubic amplitude equations. It follows that if $\mathrm{V} 1$ is not sitting close to a bifurcation point, so that much stronger modulations are needed to drive it to an orientation-tuned state, then a different mathematical analysis is needed. In this respect, it is clear that the noise fluctuations described above can play an important role in keeping the network dynamics close to the bifurcation point. As recently noted by Anderson, Lampl, Gillespie, and Ferster (2000), noise fluctuations can also secure contrast invariance in the tuned response of a population of spiking neurons, which is a property we found in the uncoupled hypercolumn model (Bressloff et al., 2000) using sigmoid population equations that effectively take account of effects produced by stochastic resonance.

5.2 Divisive Inhibition. Another well-known set of studies concerns the use of recurrent inhibition to model the nearly linear behavior of simple cortical cells. Thus, Carandini et al. (1997) have explored the use of shunting or divisive inhibition provided by a pool of inhibitory neurons, to linearize neural responses and produce contrast invariance and satura- 
tion properties consistent with observations. As Majaj, Smith, and Movshon (2000) recently showed, many contextual effects can be modeled within this framework if the inhibition acts to normalize the neural response. Thus, cross-orientation suppression within the classical receptive field is easily obtained. In general, many suppression experiments can be modeled with such a mechanism, but it is more difficult to account for effects such as cross-orientation facilitation by stimuli in the nonclassical receptive field.

The idea of using division rather than subtraction to model the effects of inhibition has been around for a long time. One early mathematical use was by Grossberg (1973), who developed population equations similar to those of Wilson and Cowan $(1972,1973)$, but using divisive inhibition. An immediate problem is then to find a biophysical basis for such a mechanism. Recent work by Wielaard et al. (2001) has provided a natural solution. If one uses conductance-based models of the evolution of changes in membrane potentials in a network of integrate-and-fire neurons with recurrent excitation and inhibition and if the conductances are sufficiently large, then the steady-state voltages reached depend on both differences between excitation and inhibition, and also on ratios of conductances, in such a way as to implement divisive inhibition. One can then use such conductance models to see how linear simple cell properties can emerge from network interactions. It is the balance between excitation and inhibition that produces effective linearity.

A similar but less biophysically direct model was also recently introduced by Chance and Abbott (2000). However, they went further in devising a circuit to maintain rapid switching in the presence of critical slowing down by using a three-population model comprising one excitatory and two (divisive) inhibitory populations. This circuit is more elaborate than that of Tsodyks and Sejnowski (1995) and in fact is very close to the three-neuron circuit we have studied in this article (see Figure 1), except that we have used subtractive inhibition. But the mathematical analysis carried out in this article proceeds by first linearizing the basic equations about an equilibrium state and then carrying out various perturbation expansions. It follows that either subtractive or divisive inhibition will lead to very similar equations and conclusions. The only difference lies in the particular parameter values needed to obtain the various behaviors. Thus, our results are qualitatively similar to what can be expected from a three-neuron circuit employing divisive rather than subtractive inhibition, particularly as we deal only with steady-state behaviors in this article.

Given the framework described above, we note again that the analysis outlined in this article provides an account of both suppression and facilitation in a single model. We expect that the equivalent divisive inhibitory model will also exhibit such properties, at least if stationary tuning curves are analyzed. It remains to be seen what differences, if any, exist in the case of time-dependent responses. 
5.3 Contrast Dependence. One other benefit is provided by the threeneuron circuit described here. In various parts of the article, we have imposed contrast dependence of the lateral connectivity in a somewhat ad hoc manner simply by assuming the effective coupling coefficient $\beta$ of the lateral connections between hypercolumns to be of the form

$$
\beta=\beta_{E}-\beta^{*}(C),
$$

where $\beta_{E}$ is the excitatory coupling between hypercolumns and $C$ is the stimulus contrast. Evidently if $\beta^{*}(C)$ increases monotonically with contrast, there exists a contrast threshold at which $\beta<0$. One relatively straightforward way to achieve this is to suppose that basket cells, which receive about $20 \%$ of the signal from excitatory lateral connections (McGuire et al., 1991), have relatively high thresholds and are therefore not activated by low-contrast stimuli. In effect, this implies that the effective excitatory part of the aggregate hypercolumn receptive field is larger at low contrast (Movshon, pers. comm., 2001).

\section{Acknowledgments}

We thank Trevor Mundel for many helpful discussions. This work was supported in part by grant 96-24 from the James S. McDonnell Foundation to J. D. C. The research of P.C. B. was supported by a grant from the Leverhulme Trust. P. C. B. wishes to thank the Mathematics Department, University of Chicago, for its hospitality and support. P. C. B. and J. D. C. also thank Geoffrey Hinton and the Gatsby Computational Neurosciences Unit, University College, London, for hospitality and support.

\section{References}

Anderson, S., Lampl, I., Gillespie, D. C., \& Ferster, D. (2000). The contribution of noise to contrast invariance of orientation tuning in cat visual cortex. Science, 290 (5498), 1968-1972.

Aronson, D. G., Ermentrout, G. B., \& Kopell, N. (1990). Amplitude response of coupled oscillators. Physica D, 41, 403-449.

Ben-Yishai, R., Bar-Or, R. L., \& Sompolinsky, H. (1995). Theory of orientation tuning in visual cortex. Proc. Nat. Acad. Sci., 92, 3844-3848.

Ben-Yishai, R., Hansel, D., \& Sompolinsky, H. (1997). Traveling waves and the processing of weakly tuned inputs in a cortical network module. J. Comput. Neurosci., 4, 57-77.

Blakemore, C., \& Tobin, E. (1972). Lateral inhibition between orientation detectors in the cat's visual cortex. Exp. Brain Res., 15, 439-440.

Blasdel, G. G. (1992). Orientation selectivity, preference, and continuity in monkey striate cortex. J. Neurosci., 12, 3139-3161.

Blasdel, G. G., \& Salama, G. (1986). Voltage-sensitive dyes reveal a modular organization in monkey striate cortex. Nature, 321, 579-585. 
Bonhoeffer, T., Kim, D., Malonek, D., Shoham, D., \& Grinvald, A. (1995). Optical imaging of the layout of functional domains in area $17 / 18$ border in cat visual cortex. European J. Neurosci., 7(9), 1973-1988.

Bosking, W. H., Zhang, Y., Schofield, B., \& Fitzpatrick, D. (1997). Orientation selectivity and the arrangemen t of horizontal connections in tree shrew striate cortex. J. Neurosci., 17, 2112-2127.

Bressloff, P. C., Bressloff, N. W., \& Cowan, J. D. (2000). Dynamical mechanism for sharp orientation tuning in an integrate-and-fire model of a cortical hypercolumn. Neural Comput., 12, 2473-2511.

Bressloff, P. C., Cowan, J. D., Golubitsky, M., Thomas, P. J., \& Wiener, M. (2001a). Geometric visual hallucinations, Euclidean symmetry and the functional architecture of striate cortex. Phil. Trans. Roy. Soc. Lond. B, 356, 299-330.

Bressloff, P. C., Cowan, J. D., Golubitsky, M., Thomas, P. J., \& Wiener, M. (2001b). What geometric visual hallucinations tell us about the visual cortex. Neural Comput., 13, 1-19.

Carandini, M., Heeger, D. J., \& Movshon, J. A. (1997). Linearity and normalization in simple cells of the macaque primary visual cortex. J. Neurosci., 17(21), 8621-8644.

Chance, F. S., \& Abbott, L. F. (2000). Divisive inhibition in recurrent networks. Network: Comp. Neural Sys., 11, 119-129.

Cowan, J. D. (1997). Neurodynamics and brain mechanisms. In M. Ito, Y. Miyashita, \& E. T. Rolls (Eds.), Cognition, computation, and consciousness (pp. 205-233). Oxford: Oxford University Press.

Cowan, J. D., \& Ermentrout, G. B. (1978). Some aspects of the "eigenbehavior" of neural nets. In S. Levin (Ed.), Studies in mathematical biology, 15 (pp. 67-117). Providence, RI: Mathematical Association of America.

DeAngelis, G., Robson, J., Ohzawa, I., \& Freeman, R. (1992). Organization of suppression in receptive fields of neurons in cat visual cortex. J. Neurophysiol., 68(1), 144-163.

Douglas, R., Koch, C., Mahowald, M., Martin, K., \& Suarez, H. (1995). Recurrent excitation in neocortical circuits. Science, 269, 981-985.

Dragoi, V., \& Sur, M. (2000). Some properties of recurrent inhibition in primary visual cortex: Contrast and orientation dependence on contextual effects. $J$. Neurophysiol., 83, 1019-1030.

Ermentrout, G. B. (1998). Neural networks as spatial pattern forming systems. Rep. Prog. Phys., 61, 353-430.

Ermentrout, G. B., \& Cowan, J. D. (1979). A mathematical theory of visual hallucination patterns. Biol. Cybernetics, 34, 137-150.

Ferster, D., Chung, S., \& Wheat, H. (1997). Orientation selectivity of thalamic input to simple cells of cat visual cortex. Nature, 380, 249-281.

Fitzpatrick, D. (2000). Seeing beyond the receptive field in primary visual cortex. Curr. Op. in Neurobiol., 10, 438-443.

Fitzpatrick, D., Zhang, Y., Schofield, B., \& Muly, M. (1993). Orientation selectivity and the topographic organization of horizontal connections in striate cortex. Soc. Neurosci. Abstracts, 19, 424.

Gilbert, C., Das, A., Ito, M., Kapadia, M., \& Westheimer, G. (1996). Spatial integration and cortical dynamics. Proc. Nat. Acad. Sci., 93, 615-622. 
Gilbert, C., \& Wiesel, T. (1983). Clustered intrinsic connections in cat visual cortex. J. Neurosci., 3, 1116-1133.

Gilbert, C., \& Wiesel, T. (1989). Columnar specificity of intrinsic horizontal and corticocortical connections in cat visual cortex. J. Neurosci., 9, 2432-2442.

Gray, C. M., Konig, P., Engel, A. K., \& Singer, W. (1989). Oscillatory responses in cat visual cortex exhibit inter-columnar synchronization which reflects global stimulus properties. Nature, 338, 334-337.

Grinvald, A., Lieke, E., Frostig, R., \& Hildesheim, R. (1994). Cortical pointspread function and long-range lateral interactions revealed by real-time optical imaging of macaque monkey primary visual cortex. J. Neurosci., 14, 2545-2568.

Grossberg, S. (1973). Contour enhancement, short term memory, and constancies in reverberating neural networks. Studies in Applied Mathematics, 52(3), 213-257.

Grossberg, S., \& Raizada, R. D. S. (2000). Contrast-sensitive perceptual grouping and object-based attention in the laminar circuits of primary visual cortex. Vision Res., 40, 1413-1432.

Hata, Y., Tsumoto, T., Sato, H., Hagihara, K., \& Tamura, H. (1988). Inhibition contributes to orientation selectivity in visual cortex of cat. Nature, 335, 815-817.

Hirsch, J., \& Gilbert, C. (1992). Synaptic physiology of horizontal connections in the cat's visual cortex. J. Physiol. Lond., 160, 106-154.

Hoppensteadt, F., \& Izhikevich, E. (1997). Weakly connected neural networks. New York: Springer.

Hubel, D., \& Wiesel, T. (1962). Receptive fields, binocular interaction and functional architecture in the cat's visual cortex. J. Neurosci., 3, 1116-1133.

Jagadeesh, B., \& Ferster, D. (1990). Receptive field lengths in cat striate cortex can increase with decreasing stimulus contrast. Soc. Neurosci. Abstr., 16, 130.11.

Kath, W. L. (1981). Resonance in periodically perturbed Hopf bifurcation. Stud. Appl. Math., 65, 95-112.

Levitt, J. B., \& Lund, J. (1997). Contrast dependence of contextual effects in primate visual cortex. Nature, 387, 73-76.

Li, C., \& Li, W. (1994). Extensive integration field beyond the classical receptive field of cat's striate cortical neurons-classification and tuning properties. Vision Res., 34(18), 2337-2355.

Li, Z. (1999). Pre-attentive segmentation in the primary visual cortex. Spatial Vision, 13, 25-39.

Majaj, N., Smith, M. A., \& Movshon, J. A. (2000). Contrast gain control in macaque area MT. Soc. Neuroci. Abstr.

Malach, R., Amir, Y., Harel, M., \& Grinvald, A. (1993). Relationship between intrinsic connections and functional architecture revealed by optical imaging and in vivo targeted biocytin injections in primate striate cortex. Proc. Natl. Acad. Sci., 90, 10469-10473.

McGuire, B., Gilbert, C., Rivlin, P., \& Wiesel, T. (1991). Targets of horizontal connections in macaque primary visual cortex. J. Comp. Neurol., 305, 370-392.

McLaughlin, D., Shapley, R., Shelley, M., \& Wielaard, D. J. (2000). A neuronal network model of macaque primary visual cortex (V1): Orientation tuning and dynamics in the input layer 4C $\alpha$. Proc. Natl. Acad. Sci., 97, 8087-8092. 
Michalski, A., Gerstein, G., Czarkowska, J., \& Tarnecki, R. (1983). Interactions between cat striate cortex neurons. Exp. Brain Res., 53, 97-107.

Mundel, T. (1996). A theory of cortical edge detection. Unpublished doctoral dissertation, University of Chicago.

Mundel, T., Dimitrov, A., \& Cowan, J. D. (1997). Visual cortex circuitry and orientation tuning. In M. C. Mozer, M. I. Jordan, \& T. Petsche(Eds.), Advances in neural information processing systems, 9 (pp. 886-893). Cambridge, MA: MIT Press.

Obermayer, K., \& Blasdel, G. (1993). Geometry of orientation and ocular dominance columns in monkey striate cortex. J. Neurosci., 13, 4114-4129.

Phleger, B., \& Bonds, A. B. (1995). Dynamic differentiation of $\mathrm{GABA}_{A}-$ sensitive influences on orientation selectivity of complex cells in the cat striate cortex. Exp. Brain Res., 104, 81-88.

Polat, U., Mizobe, K., Pettet, M. W., Kasamatsu, T., \& Norcia, A. M. (1998). Collinear stimuli regulate visual responses depending on a cell's contrast threshold. Nature, 391, 580-584.

Pugh, M. C., Ringach, D. L., Shapley, R., \& Shelley, M. J. (2000). Computational modeling of orientation tuning dynamics in monkey primary visual cortex. J. Comput. Neurosci., 8, 143-159.

Rockland, K. S., \& Lund, J. (1983). Intrinsic laminar lattice connections in primate visual cortex. J. Comp. Neurol., 216, 303-318.

Sceniak, M. P., Ringach, D. L., Hawken, M. J., \& Shapley, R. (1999). Contrast's effect on spatial summation by macaque V1 neurons. Nature Neurosci., 2, 733-739.

Sillito, A. M. (1975). The contribution of inhibitory mechanisms to the receptivefield properties of neurones in the striate cortex of the cat. J. Physiol. Lond., 250, 305-329.

Sillito, A. M., Grieve, K. L., Jones, H. E., Cudeiro, J., \& Davis, J. (1995). Visual cortical mechanisms detecting focal orientation discontinuities. Nature, 378 , 492-496.

Singer, W., \& Gray, C. M. (1995). Visual feature integration and the temporal correlation hypothesis. Ann. Rev. of Neurosci., 18, 555-586.

Somers, D., Nelson, S., \& Sur, M. (1994). Effects of long-range connections on gain control in an emergent model of visual cortical orientation selectivity. Soc. Neurosci. Abstr., 20, 646.7.

Somers, D., Nelson, S., \& Sur, M. (1995). An emergent model of orientation selectivity in cat visual cortical simple cells. J. Neurosci., 15, 5448-5465.

Somers, D., Todorov, E. V., Siapas, A. G., Toth, L. J., Kim, D.-S., \& Sur, M. (1998). A local circuit approach to understanding integration of long-range inputs in primary visual cortex. Cerebral Cortex, 8, 204-217.

Stetter, M., Bartsch, H., \& Obermayer, K. (2000). A mean field model for orientation tuning, contrast saturation, and contextual effects in the primary visual cortex. Biol. Cybernetics, 82, 291-304.

Toth, L. J., Rao, S. C., Kim, D., Somers, D., \& Sur, M. (1996). Subthreshold facilitation and suppression in primary visual cortex revealed by intrinsic signal imaging. Proc. Natl. Acad. Sci., 93, 9869-9874.

Tsodyks, M., \& Sejnowski, T. (1995). Rapid state switching in balanced cortical network models. Network: Comp. Neural Sys., 6, 1-14. 
Vidyasagar, T. R., Pei, X., \& Volgushev, M. (1996). Multiple mechanisms underlying the orientation selectivity of visual cortical neurons. TINS, 19(7), 272-277.

Walker, G. A., Ohzawa, I., \& Freeman, R. D. (1999). Asymmetric suppression outside the classical receptive field of the visual cortex. J. Neurosci., 19, 1053610553.

Wenderoth, P., \& Johnstone, S. (1988). The different mechanisms of the direct and indirect tilt illusions. Vision Res., 28, 301-312.

Wielaard, D., Shelley, M., McLaughlin, D., \& Shapley, R. (2001). How simple cells are made in a nonlinear network model of the visual cortex. J. Neurosci., 21, 5203-5211.

Wiener, M. C. (1994). Hallucinations, symmetry, and the structure of primary visual cortex: A bifurcation theory approach. Unpublished doctoral dissertation, University of Chicago.

Wilson, H. R., \& Cowan, J. D. (1972). Excitatory and inhibitory interactions in localized populations of model neurons. Biophys. J., 12, 1-24.

Wilson, H. R., \& Cowan, J. D. (1973). A mathematical theory of the functional dynamics of cortical and thalamic nervous tissue. Kybernetik, 13, 55-80.

Yoshioka, T., Blasdel, G. G., Levitt, J. B., \& Lund, J. (1996). Relation between patterns of intrinsic lateral connectivity, ocular dominance, and cytochrome oxidase-reactive regions in macaque monkey striate cortex. Cerebral Cortex, 6, 297-310.

Received January 10, 2001; accepted May 25, 2001. 